\title{
Analysis of premixed flame propagation between two closely-spaced parallel plates
}

\author{
Daniel Fernández-Galisteo ${ }^{\mathrm{a}, *}$, Vadim N. Kurdyumov ${ }^{\mathrm{a}}$, Paul D. Ronney ${ }^{\mathrm{b}}$ \\ ${ }^{a}$ Department of Energy, CIEMAT. Avda. Complutense 40, 28040 Madrid, Spain. \\ ${ }^{b}$ Department of Aerospace and Mechanical Engineering, University of Southern California, Los Angeles, \\ CA, USA.
}

\begin{abstract}
Motivated by experimental observations on premixed-gas flame propagation in Hele-Shaw cells, this work analyzes quasi-isobaric flame propagation between two adiabatic parallel plates using a simple quasi-2D formulation based on averaging the flow properties across the cell gap. Instabilities associated with thermal expansion, buoyancy, viscosity change across the front and differential diffusion of thermal energy and reactants are investigated with one-step chemistry, constant heat capacity and variable transport coefficients through time-dependent computations of the flame front evolution in large domains. These instabilities are found to induce flame wrinkling which increases flame surface area and thus propagation speeds in ways different from those associated with freely propagating flames. The simulations are compared with experiments in Hele-Shaw cells; very good qualitative and (in some cases) quantitative agreement is found.
\end{abstract}

\section{Introduction}

The propagation of premixed flames in narrow channels, tubes, gaps or slots is relevant to several areas of the combustion research, for example in internal combustion engines (ICEs) in the narrow gap between the piston and cylinder walls (called the "crevice volume") which is of relevance to the study of hydrocarbon emissions due to partial burning or flame quenching. In fact, even in the main combustion chamber of premixed-charge (e.g., gasolinefueled) ICEs, at the time of combustion the chamber has a high aspect ratio, typically 1 $\mathrm{cm}$ in height and $10 \mathrm{~cm}$ in diameter. Additionally, emerging interest for the development of portable power generation devices has led to increased interest in combustion in confined geometries with small gaps (i.e., comparable to the quenching distance) and high aspect ratios $[1,2,3,4,5,6]$.

\footnotetext{
${ }^{*}$ Corresponding author

Email address: d.galisteo@ciemat.es (Daniel Fernández-Galisteo)

Published Journal Article available online: https://doi.org/10.1016/j. combustflame.2017.11.022
} 
Significant progress in the fundamental understanding of freely propagating flames in narrow channels and ducts has been made. Experimental works have demonstrated the feasibility of combustion in microchannels [7] and numerous methods for sustaining microcombustion have been proposed [8]. Numerical simulations in the framework of the constantdensity approximation $[9,10,11,12,13,14]$ and variable density $[15,16,17,18]$ with reduced $[19,20]$ and detailed chemistry $[21,22]$ have systematically separated and studied effects as the channel size, thermal expansion, heat loss, differential diffusion, radical quenching and detailed kinetics on the flame shape and dynamics in channels whose length (in the direction of flame propagation) is much greater than its width in the two transverse dimensions.

A related but distinct configuration is the Hele-Shaw cell [23, 24] between two parallel plates separated by a narrow gap, i.e., a domain that is much larger in two dimensions than the third one. Hele-Shaw cells can be employed to study flame propagation in the manner shown in Fig. 1. In this configuration several types of flame instabilities may manifest themselves including

- The Darrieus-Landau (DL) instability $[25,26]$ due to the density change across the flame front. DL effects do not depend on the flame structure and have no characteristic length scale. DL effects occur for any propagating front in which the density of the products is lower than that of the reactants, which is the case for essentially all flames. The impact of the DL mechanism is characterized by the ratio $\rho_{u} / \rho_{b}$, where $\rho_{u}$ and $\rho_{b}$ are the densities of the fresh and burned gas mixture, respectively.

- The Rayleigh-Taylor (RT) instability [27] due to buoyant convection. RT effects do not depend on the flame structure and affect larger scales more than smaller scales. RT is destabilizing for upward-propagating flames, for which the lower-density fluid lies underneath the higher-density fluid, whereas for downward-propagating flames, RT is stabilizing. The impact of the RT mechanism is characterized by the Froude number (defined below), which is a measure of the ratio of dynamic to hydrostatic pressure.

- The Saffman-Taylor (ST) instability [28] due to the viscosity change across the flame front. ST effects do not depend on the flame structure but require confinement (e.g., in a Hele-Shaw cell) and affect larger scales more than smaller ones. ST is destabilizing when a less-viscous fluid displaces a more-viscous fluid. This is the case for essentially all flames since the viscosity of gases increases with temperature, thus burned products have higher viscosity than the unburned reactants. The impact of ST is characterized by the ratio $\mu_{b} / \mu_{u}$, where $\mu_{b}$ and $\mu_{u}$ are the dynamic viscosities of the burned and fresh gas mixtures, respectively.

- The diffusive-thermal (DT) instability [29] due to unequal values of thermal vs. molecular diffusion coefficients. This is characterized by the Lewis numbers $(L e)$ of the reactants, with $L e$ of the stoichiometrically deficient reactant having the dominant influence. DT depends on the flame structure and exhibits a maximum growth rate at a wavelength comparable to the thermal thickness of the flame $\left(\delta_{T} \equiv \mathcal{D}_{T_{u}} / S_{L}\right.$, where $S_{L}$ is the burning velocity of the planar adiabatic flame and $\mathcal{D}_{T_{u}}$ is the thermal diffusivity 
of the fresh gas mixture). DT is destabilizing for $L e$ less than a critical value near unity and is stabilizing for larger $L e$.

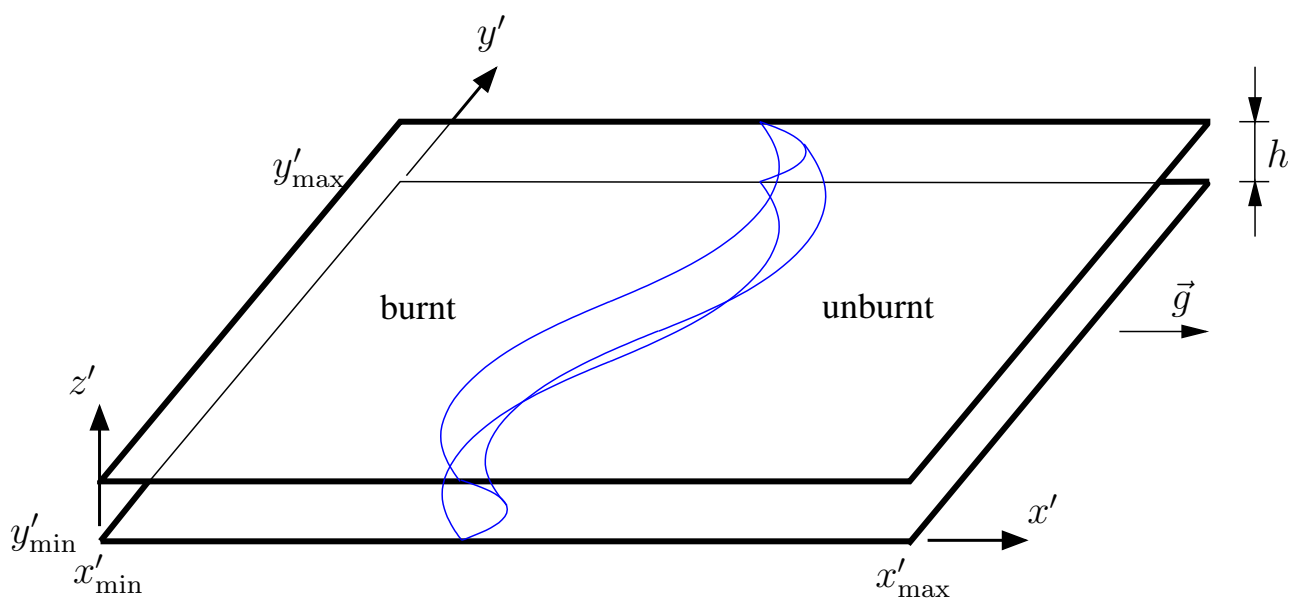

Figure 1: Sketch of the Hele-Shaw cell with a curved flame propagating from the left to the right between two parallel plates with a cell gap $h$. Shown at the right is the gravity vector, which lies along the $x$-direction. Adapted from [30].

It is anticipated that these instabilities will wrinkle the flame front and modify its overall propagation rate even though the gas flow may remain laminar. Joulin and Sivashinsky [31] performed a linear stability analysis of flame fronts in Hele-Shaw cells including DL, RT and ST (but not DT). They modeled the flame front as an infinitely thin discontinuity with specified local normal propagation speed and derived a dispersion relation expressing the growth rate of the instability in terms of the density and viscosity differences across the front and the buoyancy effect. They showed that DL and ST are unconditionally destabilizing (due to the density decrease and viscosity increase across the front) and RT is destabilizing/stabilizing for upward/downward propagation. They also showed that heat losses to the walls moderate these instability mechanisms by decreasing the density and viscosity ratios across the front.

The Joulin-Sivashinsky analysis did not include DT effects, which may be significant for Lewis numbers not close to unity. Kang et al. $[32,30]$ extended Joulin and Sivashinsky's work numerically to include DT effects using a 2D compressible reactive Navier-Stokes formulation with the Poiseuille flow assumption. These studies focused primarily on computing the linear growth rates of the aforementioned instabilities and discussing the flame shapes. Their calculations also revealed the influence of both DL and ST effects on the propagation rates. In particular, for $L e=1$ and ratios of the plate separation $h$ to the thermal flame thickness $\delta_{T}$ of the order $a \equiv h / \delta_{T}=S_{L} h / D_{T_{u}} \approx 15$, where $a$ can be interpreted as a Peclet number, the authors reported an overall flame speed $S_{T}{ }^{1}$ of about 1.4 times that of the adiabatic

\footnotetext{
${ }^{1}$ The symbol $S_{T}$ is normally associated with the propagation speed of flames in forced turbulence which
} 
planar velocity $S_{L}$. Excluding ST by neglecting the viscous drag due to Poiseuille flow, $S_{T} / S_{L}$ decreased to about 1.25 , in agreement with values previously calculated for purely 2D simulations that included DL but not ST effects [33, 34, 35, 36, 37].

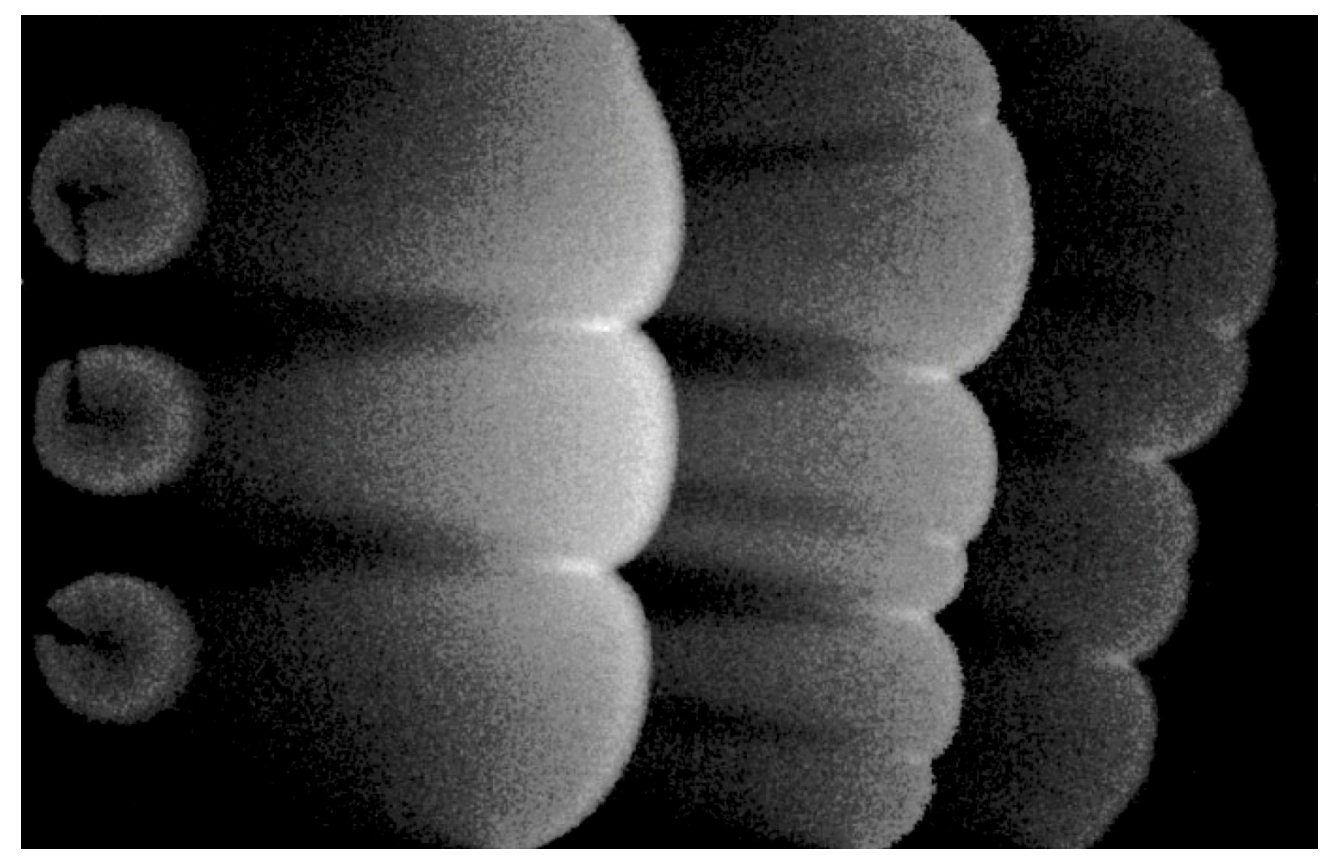

Figure 2: Sequential, superimposed images of a flame propagating horizontally from left to right in a HeleShaw cell in a $25.0 \% \mathrm{H}_{2}-6.25 \% \mathrm{O}_{2}-68.75 \% \mathrm{~N}_{2}$ mixture (calculated $S_{L}=52 \mathrm{~cm} / \mathrm{s}, \rho_{u} / \rho_{b}=4.1$ ) [38]. Field of view is $597 \mathrm{~mm} \times 394 \mathrm{~mm}$ with a cell gap $h=12.7 \mathrm{~mm}$, thus $a \approx 171$.

Experimental studies have investigated and identified the contributions of some of these intrinsic instability mechanisms in Hele-Shaw cells [39, 38, 40], making use of different mixtures, direction of propagation and distance between the plates. The image in Fig. 2 shows the wrinkled shape of a flame propagating horizontally in a rich $\mathrm{H}_{2}-\mathrm{O}_{2}-\mathrm{N}_{2}$ mixture (for which the stoichiometrically deficient reactant is $\mathrm{O}_{2}$ and the effective $L e \approx 1.6$ ). The flame is ignited by 3 electric sparks near the left side of the cell and propagates from the left (open to the atmosphere) end towards the right (closed) end, so that the flame propagates nearly isobarically into a nominally quiescent gas and thus the observed propagation speed in the laboratory frame of reference is equal to the propagation speed relative to the unburned gas $S_{T}$. As discussed later, the large wavelengths of wrinkling are caused by thermal expansion (DL) effects. In contrast, for a lean $\mathrm{H}_{2}-\mathrm{O}_{2}-\mathrm{N}_{2}$ mixture (for which the stoichiometrically deficient reactant is $\mathrm{H}_{2}$ and thus the effective $L e \approx 0.34$ ) (Fig. 3 [38]), DT influences promote the appearance of smaller cellular structures embedded into larger DL-generated wrinkles.

is absent in this work, nevertheless, we adopt this notation to signify that the self-induced flame instabilities studied here can also lead to wrinkling which accelerates propagation speeds in a manner analogous to flame wrinkling by forced turbulence. 


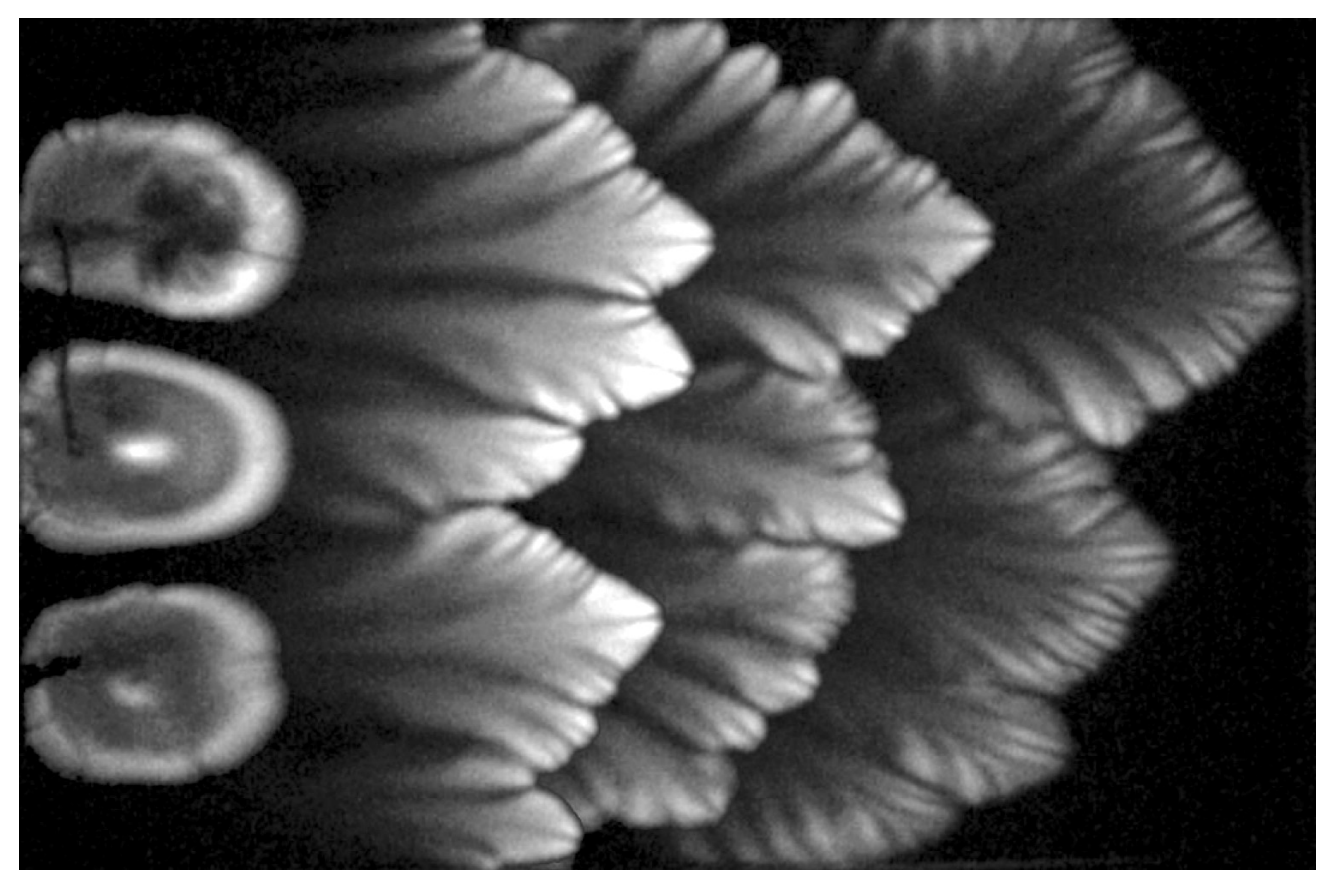

Figure 3: Sequential, superimposed images of a flame propagating horizontally from left to right in a HeleShaw cell in a $12.7 \% \mathrm{H}_{2}-7.9 \% \mathrm{O}_{2}-79.4 \% \mathrm{~N}_{2}$ mixture (calculated $S_{L}=9.7 \mathrm{~cm} / \mathrm{s}, \rho_{u} / \rho_{b}=4.1$ ) [38]. Field of view is $597 \mathrm{~mm} \times 394 \mathrm{~mm}$ with a cell gap $h=12.7 \mathrm{~mm}$, thus $a \approx 42$.

It may be noted that without the small-scale DT wrinkling, the larger-scale hydrodynamic structures are cusp-shaped with the sharp peaks pointed toward the burned gas (Fig. 2), whereas when DT instabilities are present, the large-scale structure is more angular or "sawtoothed"; this was a consistent feature of the experiments [38]. For a downward-propagating flame in a lean $\mathrm{H}_{2}-\mathrm{O}_{2}-\mathrm{N}_{2}$ mixture this angularity is retained but the overall structure is flatter due to the stabilizing RT effects at large scales (Fig. 4). In the experiments, the measured propagation rates are about $S_{T} / S_{L} \approx 2.5$ (Fig. 2), $S_{T} / S_{L} \approx 15$ (Fig. 3) and $S_{T} / S_{L} \rightarrow \infty$ (Fig. 4).

The influences of DT and RT on flame propagation in Hele-Shaw cells are readily assessed by changing the effective mixture $L e$ and propagation direction, respectively. In contrast, the relative influences of DL and ST are not as easily identified since both occur in principle for any mixture, plus the density and viscosity ratios scale similarly with temperature. While DL is unrelated to any confinement effects and thus the cell gap $h$, ST is due to viscous drag and thus will increase with decreasing $h$. Experimentally, a difficulty arises in testing the effect of $h$ on flame behavior because when the parameter $a$ is less than a critical value of about 40 flame quenching occurs due to heat losses to the walls [41]. Kang et al. [32] proposed that this critical $a$ is too large to enable observation of ST influences on flame dynamics. The situation is different for experiments in lean $\mathrm{H}_{2}-\mathrm{O}_{2}-\mathrm{N}_{2}$ mixtures [38], where DT effects locally increase the temperature in the positively curved part of the flame and, as a consequence, flames can exist as curved structures in very lean mixtures without thermal 


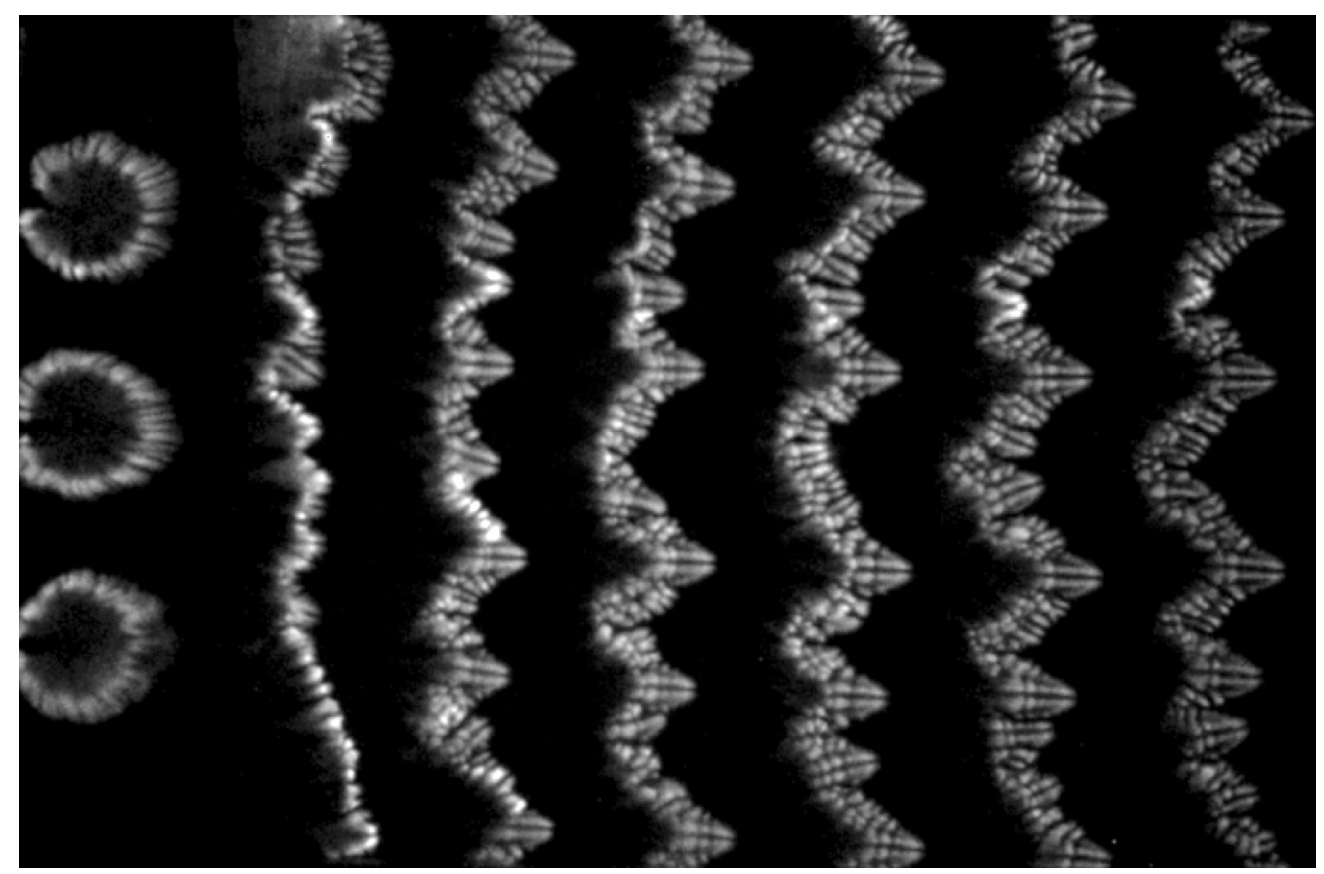

Figure 4: Sequential, superimposed images of a flame propagating downward in a Hele-Shaw cell in a $9.3 \% \mathrm{H}_{2}-13.3 \% \mathrm{O}_{2}-77.4 \% \mathrm{~N}_{2}$ mixture $\left(\rho_{u} / \rho_{b}=3.4\right)$ [38]. Field of view is $597 \mathrm{~mm} \times 394 \mathrm{~mm}$ with a cell gap $h=12.7 \mathrm{~mm}$. (For this case the calculated $S_{L}$ is extremely small, less than $0.2 \mathrm{~cm} / \mathrm{s}$, thus a meaningful value of $a$ cannot be assigned; flame propagation is possible only because of DT in this low-Le mixture.)

quenching that would not be possible in a planar configuration. In fact, for such cases the Peclet number a can be of order unity or less due to the decrease of $S_{L}$ and the increase of $\delta_{T}$ for which ST influences could be very significant. Consequently, the asymptotic limit $a \rightarrow 0$ employed in this numerical study may be justified. Sometimes the results of these limits remain surprisingly valid even for values of $a$ larger than expected (i.e., of order unity or larger [18]).

In view of the above discussion, the objectives of this study are to model flames in HeleShaw cells with a particular aim of determining the effects of the aforementioned instability mechanisms (DL, RT, ST and DT) on the flame shapes and propagation rates. By making use of the asymptotic limit $a \rightarrow 0$, we investigate numerically the relative influences of these intrinsic instabilities. The dependence of the domain size and initial perturbations on flame evolution are addressed and comparisons with experiments made where possible.

\section{Formulation and governing equations}

Consider premixed flame propagation between two parallel plates as shown in Fig. 1. We restrict the study to a semi-closed system, with the mixture being ignited at the left (open) end and propagating towards the (closed) end wall at the right, as in the aforementioned experiments. The following assumptions were made: 
1. Low Mach number approximation. The propagation speeds $S_{T}$ of the corrugated flames are much smaller than the speed of sound, thus the thermodynamic pressure is instantaneously (compared to the flame response time) equalized throughout the domain to the value $p_{\text {atm }}$ dictated by the atmospheric pressure at the open end. Deviations from $p_{\text {atm }}$ are limited to small hydrodynamic variations given by $p^{\prime}-p_{\text {atm }}$. Hereafter primes $\left({ }^{\prime}\right)$ denote dimensional quantities that depend on time and location.

2. Constant mixture heat capacity $c_{p}$ and average molecular weight.

3. Negligible Soret and Dufour effects. While it is well known that Soret diffusion does affect flame stability [42], the effect is primarily quantitative, not qualitative, i.e., it results in a decrease in the effective $L e$ via an increase in the mass diffusion coefficients [43].

4. Temperature-dependent transport properties. This assumption is essential for investigating the influence of the ST instability. Following [44] and others, we assume that viscosity follows the relation $\mu^{\prime} / \mu_{u}=\left(T^{\prime} / T_{u}\right)^{\sigma}$, where $\mu^{\prime}$ is the dynamic viscosity of the gas mixture and $\sigma$ an exponent taken to be 0 or 0.7 in this work. We also assume constant Prandtl number and $c_{p}$, thus the gas thermal conductivity $\lambda^{\prime}$ has the same temperature dependence as $\mu^{\prime}$. The effective mixture $L e$ is also assumed to be constant.

5. Single-step chemistry. Chemical reaction is modeled with a single irreversible reaction of the form $F+O \rightarrow P$, where $F, O$ and $P$ denote fuel, oxidant and products, respectively. We consider very fuel-lean mixtures so that the concentration of oxidant remains nearly constant. The chemical reaction rate (moles of fuel per unit time per unit volume) is assumed to be governed by an Arrhenius expression, first order in fuel and oxidant, given by

$$
\omega^{\prime}=\mathcal{B} \rho^{\prime 2} Y_{F} \exp \left(-E / \mathcal{R} T^{\prime}\right),
$$

where $\mathcal{B}$ is the preexponential factor containing the mass fraction of the oxidant and the molecular masses of both fuel and oxidant, $\rho^{\prime}$ is the density of the mixture, $E$ is the activation energy, $\mathcal{R}$ is the universal gas constant, $Y_{F}$ is the fuel mass fraction and $T^{\prime}$ is the temperature.

6. Adiabatic walls. The rationale and limitations of this assumption are discussed below.

With these assumptions, and following a classical approach of using the thermal flame thickness $\delta_{T}$ as the reference length scale, together with the properties of the fresh unburnt mixture for density $\rho_{u}$, temperature $T_{u}$, and fuel mass fraction $Y_{F_{u}}$, the dimensionless variables can be written as follows

$$
\begin{gathered}
(x, y, z)=\left(x^{\prime} / \delta_{T}, y^{\prime} / \delta_{T}, z^{\prime} / h\right), \quad t=t^{\prime} S_{L} / \delta_{T}, \\
(u, v, w)=\left(u^{\prime} / S_{L}, v^{\prime} / S_{L}, w^{\prime} /\left(a S_{L}\right)\right), \quad \rho=\rho^{\prime} / \rho_{u} \\
p=a^{2}\left(p^{\prime}-p_{\text {atm }}\right) /\left(12 \operatorname{Pr} \rho_{u} S_{L}^{2}\right), \quad \theta=\left(T^{\prime}-T_{u}\right) /\left(T_{a d}-T_{u}\right), \quad Y=Y_{F} / Y_{F_{u}},
\end{gathered}
$$

corresponding to values of the Cartesian coordinates, time, velocity components, density, hydrodynamic pressure variations, temperature, and fuel mass fraction, respectively. Here 
$T_{a d}=T_{u}+Q Y_{F_{u}} / c_{p}$ is the adiabatic flame temperature, where $Q$ is the heat of combustion per unit mass of fuel. As previously discussed, the Peclet number $a=h / \delta_{T}$ is the ratio of the plate separation to the thermal flame thickness. The factor $12 \operatorname{Pr}$, where $\operatorname{Pr}=\mu^{\prime} / \rho^{\prime} \mathcal{D}_{T}$ is the Prandtl number, is introduced to scale the pressure for the sake of convenience in the analysis that follows.

Introducing the above-mentioned variables, the dimensionless governing equations become

$$
\begin{aligned}
& \frac{\partial \rho}{\partial t}+\frac{\partial(\rho u)}{\partial x}+\frac{\partial(\rho v)}{\partial y}+\frac{\partial(\rho w)}{\partial z}=0 \\
& \rho\left(\frac{\partial u}{\partial t}+u \frac{\partial u}{\partial x}+v \frac{\partial u}{\partial y}+w \frac{\partial u}{\partial z}\right)=-\frac{12 \operatorname{Pr}}{a^{2}} \frac{\partial p}{\partial x}+ \\
& \operatorname{Pr}\left\{\frac{\partial}{\partial x}\left[\mu\left(\frac{4}{3} \frac{\partial u}{\partial x}-\frac{2}{3} \frac{\partial v}{\partial y}-\frac{2}{3} \frac{\partial w}{\partial z}\right)\right]+\right. \\
& \left.\frac{\partial}{\partial y}\left[\mu\left(\frac{\partial v}{\partial x}+\frac{\partial u}{\partial y}\right)\right]+\frac{\partial}{\partial z}\left[\mu\left(\frac{\partial w}{\partial x}+\frac{1}{a^{2}} \frac{\partial u}{\partial z}\right)\right]\right\}+\frac{12 \operatorname{Pr}}{a^{2}} \rho G, \\
& \rho\left(\frac{\partial v}{\partial t}+u \frac{\partial v}{\partial x}+v \frac{\partial v}{\partial y}+w \frac{\partial v}{\partial z}\right)=-\frac{12 \operatorname{Pr}}{a^{2}} \frac{\partial p}{\partial y}+\operatorname{Pr}\left\{\frac{\partial}{\partial x}\left[\mu\left(\frac{\partial v}{\partial x}+\frac{\partial u}{\partial y}\right)\right]+\right. \\
& \left.\frac{\partial}{\partial y}\left[\mu\left(-\frac{2}{3} \frac{\partial u}{\partial x}+\frac{4}{3} \frac{\partial v}{\partial y}-\frac{2}{3} \frac{\partial w}{\partial z}\right)\right]+\frac{\partial}{\partial z}\left[\mu\left(\frac{\partial w}{\partial y}+\frac{1}{a^{2}} \frac{\partial v}{\partial z}\right)\right]\right\} \\
& \rho\left(\frac{\partial w}{\partial t}+u \frac{\partial w}{\partial x}+v \frac{\partial w}{\partial y}+w \frac{\partial w}{\partial z}\right)=-\frac{12 \operatorname{Pr}}{a^{4}} \frac{\partial p}{\partial z}+ \\
& \operatorname{Pr}\left\{\frac{\partial}{\partial x}\left[\mu\left(\frac{\partial w}{\partial x}+\frac{1}{a^{2}} \frac{\partial u}{\partial z}\right)\right]+\right. \\
& \left.\frac{\partial}{\partial y}\left[\mu\left(\frac{\partial w}{\partial y}+\frac{1}{a^{2}} \frac{\partial v}{\partial z}\right)\right]+\frac{1}{a^{2}} \frac{\partial}{\partial z}\left[\mu\left(-\frac{2}{3} \frac{\partial u}{\partial x}-\frac{2}{3} \frac{\partial v}{\partial y}+\frac{4}{3} \frac{\partial w}{\partial z}\right)\right]\right\}, \\
& \rho\left(\frac{\partial \theta}{\partial t}+u \frac{\partial \theta}{\partial x}+v \frac{\partial \theta}{\partial y}+w \frac{\partial \theta}{\partial z}\right)=\frac{\partial}{\partial x}\left(\mu \frac{\partial \theta}{\partial x}\right)+\frac{\partial}{\partial y}\left(\mu \frac{\partial \theta}{\partial y}\right)+ \\
& \frac{1}{a^{2}} \frac{\partial}{\partial z}\left(\mu \frac{\partial \theta}{\partial z}\right)+\omega \\
& \rho\left(\frac{\partial Y}{\partial t}+u \frac{\partial Y}{\partial x}+v \frac{\partial Y}{\partial y}+w \frac{\partial Y}{\partial z}\right)=\frac{1}{L e}\left[\frac{\partial}{\partial x}\left(\mu \frac{\partial Y}{\partial x}\right)+\frac{\partial}{\partial y}\left(\mu \frac{\partial Y}{\partial y}\right)+\right. \\
& \left.\frac{1}{a^{2}} \frac{\partial}{\partial z}\left(\mu \frac{\partial Y}{\partial z}\right)\right]-\omega
\end{aligned}
$$


with the ideal gas equation of state

$$
\rho=1 /(1+q \theta)
$$

and the dimensionless rate of fuel consumption given by

$$
\omega=\frac{\beta^{2}}{2 s_{L}^{2} L e} \frac{\mu_{u}}{\mu_{b}}\left(\frac{\rho_{u}}{\rho_{b}}\right)^{2} \rho^{2} Y \exp \left\{\frac{\beta(\theta-1)}{1+[q /(1+q)](\theta-1)}\right\} .
$$

From this formulation, the dimensionless parameters that appear are the Zel'dovich number $\beta=E\left(T_{a d}-T_{u}\right) / \mathcal{R} T_{a d}^{2}$, the thermal expansion coefficient $q=\rho_{u} / \rho_{b}-1=\left(T_{a d}-\right.$ $\left.T_{u}\right) / T_{u}$, the Lewis number $L e$, the exponent $\sigma$ of the reduced viscosity law $\mu=\mu^{\prime} / \mu_{u}=$ $(1+q \theta)^{\sigma}$, and the buoyancy parameter $G=a^{2} g \delta_{T} /\left(12 \operatorname{Pr} S_{L}^{2}\right)$, where $G^{-1}$ can be interpreted as a Froude number based on flame thickness $\delta_{T}$ and burning velocity $S_{L}$, scaled by $12 \mathrm{Pr} / \mathrm{a}^{2}$. With the sign convention we adopted (Fig. 1), positive $G$ corresponds to downward flame propagation in the $x$-direction and negative $G$ corresponds to upward propagation. Values of $\beta=10, q=5$ and $\sigma=0$ or 0.7 will be employed as representative of laboratory flames, except where indicated otherwise.

The factor $s_{L}=S_{L} /\left(S_{L}\right)_{\text {asp }}$ corresponds to the eigenvalue of the steady planar adiabatic problem

$$
\begin{aligned}
& -\frac{\mathrm{d} \theta}{\mathrm{d} \xi}=\frac{\mathrm{d}}{\mathrm{d} \xi}\left(\mu \frac{\mathrm{d} \theta}{\mathrm{d} \xi}\right)+\omega, \quad-\frac{\mathrm{d} Y}{\mathrm{~d} \xi}=\frac{1}{L e} \frac{\mathrm{d}}{\mathrm{d} \xi}\left(\mu \frac{\mathrm{d} Y}{\mathrm{~d} \xi}\right)-\omega, \\
& \xi \rightarrow \infty: \theta=Y-1=0, \quad \xi \rightarrow-\infty: \mathrm{d} \theta / \mathrm{d} x=\mathrm{d} Y / \mathrm{d} \xi=0,
\end{aligned}
$$

to be calculated in advance, with $\omega$ given by Eq. (9). This factor ensures that the propagation velocity of the planar front equals one during our computations. The approximate expression for the laminar flame speed, valid for $\beta \rightarrow \infty$, is given by $\left(S_{L}\right)_{a s p}=$ $\sqrt{2 L e \mathcal{B} \rho_{b} \mathcal{D}_{T_{b}} / \beta^{2}}\left(\rho_{b} / \rho_{u}\right) \exp \left(-E / 2 \mathcal{R} T_{a d}\right)$, where $\mathcal{D}_{T_{b}}$ is the thermal diffusivity of the burned gas mixture.

No-slip, non-porous and adiabatic conditions are assumed at the plate surfaces $z=0$ and $z=1$, thus the relevant boundary conditions are

$$
u=v=w=\frac{\partial \theta}{\partial z}=\frac{\partial Y}{\partial z}=0 .
$$

The boundary conditions imposed at the ends of the plates, i.e., at $x=x_{\min }, x=x_{\max }$, $y=y_{\min }$, and $y=y_{\max }$ will be discussed in the next section.

\section{The limit of closely spaced parallel plates, $a \rightarrow 0$}

In the limit where the plates are sufficiently close together so that viscous losses to the plates is significant, one can expand all variables in powers of $a^{2}$, according to $f=$ $f_{0}+a^{2} f_{1}+\mathcal{O}\left(a^{4}\right)$, where $f$ stands for the temperature $\theta$, mass fraction $Y$, density $\rho$, pressure $p$, and the velocity components $u, v$, and $w$. The adopted approach is an extension of recent studies based on the limit of narrow channels or thick flames [9, 15, 16, 18, 45, 17]. 
To leading order, Eqs. (6) and (7) yield

$$
\frac{\partial}{\partial z}\left(\mu\left(\theta_{0}\right) \frac{\partial Y_{0}}{\partial z}\right)=\frac{\partial}{\partial z}\left(\mu\left(\theta_{0}\right) \frac{\partial \theta_{0}}{\partial z}\right)=0
$$

which, when integrating with respect to the transverse coordinate $z$, using the boundary conditions $(10)$, lead to $Y_{0}=Y_{0}(x, y ; t)$ and $\theta_{0}=\theta_{0}(x, y ; t)$.

The convection of momentum terms becomes negligible to leading order in the momentum equations (3)-(5), yielding

$$
\frac{\partial p_{0}}{\partial z}=0, \quad \nabla p_{0}=\frac{\mu}{12} \frac{\partial^{2} \vec{u}_{0}}{\partial z^{2}}+\rho_{0} G \vec{e}_{x},
$$

where $\vec{u}_{0}=u_{0} \vec{e}_{x}+v_{0} \vec{e}_{y}$. Eqs. (12) give $p_{0}=p_{0}(x, y ; t)$ and $\vec{u}_{0}=6 \vec{U} z(1-z)$, where $\vec{U}=U_{x} \vec{e}_{x}+U_{y} \vec{e}_{y}$ is the corresponding $z$-averaged velocity, given by

$$
\vec{U}(x, y ; t)=\left(\int_{0}^{1} u_{0} \mathrm{~d} z\right) \vec{e}_{x}+\left(\int_{0}^{1} v_{0} \mathrm{~d} z\right) \vec{e}_{y}=-\frac{1}{\mu}\left(\nabla p_{0}-\rho_{0} G \vec{e}_{x}\right),
$$

which indicates that the flow between the plates is governed by Darcy's law. It may be noted from Eq. (13) that buoyancy can compete with the viscosity-induced pressure gradient if $G>0$. This will result in stabilizing effects of the flame-front [31], as discussed below.

The continuity equation (2) can be integrated along the $z$ coordinate making use of the boundary condition (10) at $z=0$ to yield

$$
w_{0}=-\frac{1}{\rho_{0}}\left[\frac{\partial \rho_{0}}{\partial t} z+\frac{\partial\left(\rho_{0} U_{x}\right)}{\partial x}\left(3 z^{2}-2 z^{3}\right)+\frac{\partial\left(\rho_{0} U_{y}\right)}{\partial y}\left(3 z^{2}-2 z^{3}\right)\right] .
$$

The boundary condition (10) at $z=1$ in Eq. (14) implies that

$$
\frac{\partial \rho_{0}}{\partial t}+\frac{\partial\left(\rho_{0} U_{x}\right)}{\partial x}+\frac{\partial\left(\rho_{0} U_{y}\right)}{\partial y}=0,
$$

which is finally used to yield the expression for the transverse velocity between the plates

$$
w_{0}(x, y, z ; t)=-\frac{1}{\rho_{0}} \frac{\partial \rho_{0}}{\partial t}\left(2 z^{3}-3 z^{2}+z\right) .
$$

To determine $\theta_{0}$ and $Y_{0}$, the next order of Eqs. (6) and (7) is considered:

$$
\begin{aligned}
\frac{\partial}{\partial z}\left(\mu\left(\theta_{0}\right) \frac{\partial \theta_{1}}{\partial z}\right)=\rho_{0}\left(\frac{\partial \theta_{0}}{\partial t}+u_{0} \frac{\partial \theta_{0}}{\partial x}+v_{0} \frac{\partial \theta_{0}}{\partial y}\right)- \\
\frac{\partial}{\partial x}\left(\mu\left(\theta_{0}\right) \frac{\partial \theta_{0}}{\partial x}\right)-\frac{\partial}{\partial y}\left(\mu\left(\theta_{0}\right) \frac{\partial \theta_{0}}{\partial y}\right)-\omega\left(\theta_{0}, Y_{0}\right),
\end{aligned}
$$




$$
\begin{aligned}
\frac{1}{L e} \frac{\partial}{\partial z}\left(\mu\left(\theta_{0}\right) \frac{\partial Y_{1}}{\partial z}\right)=\rho_{0}\left(\frac{\partial Y_{0}}{\partial t}+\right. & \left.u_{0} \frac{\partial Y_{0}}{\partial x}+v_{0} \frac{\partial Y_{0}}{\partial y}\right)- \\
& \frac{1}{L e}\left[\frac{\partial}{\partial x}\left(\mu\left(\theta_{0}\right) \frac{\partial Y_{0}}{\partial x}\right)+\frac{\partial}{\partial y}\left(\mu\left(\theta_{0}\right) \frac{\partial Y_{0}}{\partial y}\right)\right]+\omega\left(\theta_{0}, Y_{0}\right) .
\end{aligned}
$$

Integrating the above equations across the $z$ coordinate and using the boundary conditions (10) yields the following conservation equations for the temperature and the fuel mass fraction

$$
\begin{gathered}
\rho \frac{\partial \theta}{\partial t}+\rho U_{x} \frac{\partial \theta}{\partial x}+\rho U_{y} \frac{\partial \theta}{\partial y}=\nabla \cdot(\mu \nabla \theta)+\omega \\
\rho \frac{\partial Y}{\partial t}+\rho U_{x} \frac{\partial Y}{\partial x}+\rho U_{y} \frac{\partial Y}{\partial y}=\frac{1}{L e} \nabla \cdot(\mu \nabla Y)-\omega
\end{gathered}
$$

where the subscript 0 is dropped from now on and the symbol s stands for the dot product.

Finally, by adding Eqs. (15) and (18) after multiplying the latter by $q \rho$, and using the equation of state (8) one finds that

$$
\frac{\partial U_{x}}{\partial x}+\frac{\partial U_{y}}{\partial y}=q[\nabla \cdot(\mu \nabla \theta)+\omega]
$$

Using now (8) and (13) in (20), the following Poisson's equation for the hydrodynamic pressure field is obtained

$$
\nabla^{2} p=G \frac{\partial \rho}{\partial x}+\frac{1}{\mu}\left(\nabla p \cdot \nabla \mu-\rho G \frac{\partial \mu}{\partial x}\right)-q \mu[\nabla \cdot(\mu \nabla \theta)+\omega]
$$

where $\nabla^{2}$ is the Laplace operator written here in the $(x, y)$ plane. Eq. (21) indicates that changes in the dynamic pressure are associated with 1) density gradients when gravity is present, 2) viscosity gradients and 3) temperature gradients.

At leading order, the temperature, fuel mass fraction and velocity fields are finally obtained by solving Eqs. (8), (13), (18), (19), and (21) together with the following periodic boundary conditions in the lateral $(y)$ direction

$$
\begin{aligned}
\theta\left(x, y_{\min }\right) & =\theta\left(x, y_{\max }\right), & Y\left(x, y_{\min }\right) & =Y\left(x, y_{\max }\right) \\
\partial \theta\left(x, y_{\min }\right) / \partial y & =\partial \theta\left(x, y_{\max }\right) / \partial y, & \partial Y\left(x, y_{\min }\right) / \partial y & =\partial Y\left(x, y_{\max }\right) / \partial y \\
U_{x}\left(x, y_{\min }\right) & =U_{x}\left(x, y_{\max }\right), & U_{y}\left(x, y_{\min }\right) & =U_{y}\left(x, y_{\max }\right) .
\end{aligned}
$$

At the left side, the pressure is taken as constant and equal to the ambient pressure and the state of the mixture is assumed uniform:

$$
x=x_{\min }: \quad p=\partial^{2} \theta / \partial^{2} x=\partial^{2} Y / \partial^{2} x=0 .
$$

The boundary conditions at the left side for the temperature and mass fraction exclude the ignition event and replaces the zero-gradient condition which should be imposed when the 
flame moves far downstream from the left boundary. At the right side, zero velocity was imposed, together with uniformity of the state of the mixture:

$$
x=x_{\max }: \quad U_{x}=\partial \theta / \partial x=\partial Y / \partial x=0 .
$$

The slip condition for $U_{y}$ is allowed in the present limit due to the order reduction of the momentum equations. A thorough analysis shall include the study of the wall boundary layer at $x=x_{\max }$, but this effect can be neglected at first approximation as long as the flame remains an $O\left(\delta_{T}\right)$ distance away from the right boundary.

\section{Numerical treatment}

Time-dependent computations were conducted using the explicit MacCormack scheme, with second-order temporal accuracy. The time step was taken as $\triangle_{t}=2 \cdot 10^{-5}$, with $\triangle_{t}=$ $10^{-4}$ the maximum value satisfying the CFL condition. At every time step the aggregationbased algebraic multigrid method described in [46, 47, 48, 49] was used to solve the Poissonlike Eq. (21). The spatial derivatives of the governing equations were discretized using a second-order, three-point central difference method on a uniform rectangular grid. Following Sharpe et al. [50], who modeled freely propagating flames, we assessed the impact of grid resolution on our results by testing three different resolutions $\Delta_{x}=\Delta_{y}=0.2,0.1$ and 0.05, corresponding approximately to 4,8 and 16 grid points within the reaction zone, respectively. Sharpe et al. performed a thorough study of the grid and temporal resolutions necessary to capture the evolution of flame shape, emphasizing the importance of adequate resolution to interpret the physical results correctly. We made a similar assessment for our confined flames and came to a similar conclusion, the results of which are given apart in Supplementary materials. Here we summarize those results by stating that a resolution of 16 grid points within the flame thickness $\delta_{f}$ (defined below) was required to adequately capture the flame dynamics and propagation rates, i.e., smaller grids did not result in a substantive change in the results and coarser grids showed an incorrect evolution and final state of the flame shapes, specifically for computational domains of increasing width in the $y$ direction.

Table 1 shows that the flame thickness computed with variable viscosity is larger than that with the constant-viscosity model $(\sigma=0)$ so the flame shape structures plotted in the sections below should actually be rescaled with the more realistic definition of the flame thickness $\delta_{f}=\left(T_{a d}-T_{0}\right) / \max (\partial T / \partial x)$, as was done by [32]. However, the value of overall propagation rate scaled with the planar flame velocity $S_{T} / S_{L}$ can be directly compared without further normalization of the length and time scales. Table 1 gives the relation between $\delta_{f}$ and $\delta_{T}$ in a planar flame with one-step kinetics (1). Note that in the case of constant viscosity, the two definitions of flame thickness, $\delta_{T}$ and $\delta_{f}$, yield similar results.

The numerical method we employed is straightforward to implement but computationally expensive due to the total number of calls made to the multigrid Poisson solver. Computations were performed on a Linux cluster employing 64 processors running with a clock speed of $3.0 \mathrm{GHz}$, requiring up to $500 \mathrm{hr}$ to capture the complete flame front evolution in the largest computational domain $(400 \times 100)$. 
Table 1: Ratio of $\delta_{f}$ to the thermal flame thickness $\delta_{T}$ in a one-dimensional computation using one-step kinetics, with $\beta=10$.

\begin{tabular}{cccc}
\hline$L e$ & $q$ & $\sigma$ & $\delta_{f} / \delta_{T}$ \\
\hline 1 & 5 & 0 & 1.43 \\
1 & 5 & 0.7 & 4.33 \\
0.3 & 3 & 0 & 1.35 \\
0.3 & 3 & 0.7 & 3.12 \\
\hline
\end{tabular}

\section{Simulation results}

An important product of the solution of the governing equations formulated above is the overall propagation rate $S_{T}$ of the flame front. In the case of steady propagation the problem is translationally invariant and Eq. (19) can be integrated in a reference frame moving at $S_{T}$ using the periodic boundary conditions (22) with complete combustion behind the flame, to obtain [9]

$$
\frac{S_{T}}{S_{L}}=\frac{1}{L_{y}} \int_{x_{\min }}^{x_{\max }} \int_{y_{\min }}^{y_{\max }} \omega \mathrm{d} x \mathrm{~d} y
$$

where $L_{y}=y_{\max }-y_{\min }$ is the lateral width of the computational domain. In what follows, the value of $S_{T} / S_{L}$ calculated from (25) will be employed to characterize the flame front propagation velocity, although it is not strictly valid during unsteady propagation.

In the computations, the ignition of the mixture is simulated at $t=0$ with a series of $N$ evenly-spaced hot spots for the temperature and a uniform distribution for the fuel mass fraction, i.e.,

$$
t=0: \quad \theta=\theta_{s} \sum_{i=1}^{N} \exp \left\{\left[x^{2}-\left(y-y_{i_{s}}\right)^{2}\right] / r_{s}^{2}\right\} \quad \text { and } \quad Y=1
$$

where $y_{i_{s}}$ corresponds to the location of the $i$ th hot spot, with $r_{s}$ and $\theta_{s}$ the radius and the peak temperature, respectively. Typical values used were $\theta_{s}=1.4, r_{s}=1$ and $y_{i_{s}}=$ $L_{y}(i-1) /(N-1)$ for $i=1, \ldots, N$, with $N \neq 1$. In the first stage of propagation the $N$ curved flame fronts separated by a distance $d_{s}=L_{y} /(N-1)$ grow radially and accelerate due to thermal expansion before they merge into a roughly planar flame, as shown in Fig. 5. During the second stage the planar flame front becomes unstable and a number of wavelengths of instability are accommodated within the domain, following cell splitting and/or merging to finally reach a nearly steady propagation speed which is larger than that of the planar flame. For Le close to or larger than unity, the flame wrinkles eventually coalesce into a single-cusp structure (sometimes called a "tulip flame") except for the small lateral domain sizes, e.g., $L_{y}=12.5$ where the flame exhibits a planar shape as expected. For sufficiently small $L e$, the flame structure is continually modified by the spontaneous formation of small cellular structures which increase the propagation speed relative to that of higher Le cases. 
To better characterize the nonlinear evolution of the propagating problem, we divide the results of the simulations into six subsections, organized as follows. The first two subsections 5.1 and 5.2 are focused on the effect of physical conditions for a baseline case, namely, the lateral domain width $\left(L_{y}\right)$ and the initial perturbation scale $\left(d_{s}\right)$. The remaining subsections 5.4 to 5.6 examine the dependence of the flame dynamics and propagation rates on the $\mathrm{ST}, \mathrm{DL}, \mathrm{RT}$, and DT instability mechanisms by varying the parameters $\sigma, q, G$, and $L e$, respectively.

\subsection{Influence of the lateral domain width}

Computations of 2D freely propagating flames in periodic domains subject to DL instabilities $[33,36,34,35]$ have shown that the overall propagation rate depends on the lateral domain width $L_{y}$ but becomes independent of $L_{y}$ for sufficiently large values. In this limit, the flames evolve to a configuration consisting of a single cusp-like structure, i.e. the "tulip flame". To determine if similar behavior occurs in our highly confined flames, we performed a set of computations with a set of fixed "baseline" conditions ( $L e=1, \beta=10, q=5$, $G=0$, and $\sigma=0$ ) but varying $L_{y}$. The large number of grid points needed to capture flame evolution restricts our computational domains to $L_{y}$ no larger than 100 at full resolution $\Delta_{x}=\Delta_{y}=0.05$ and $L_{y}$ no larger than 200 at reduced resolution $\Delta_{x}=\Delta_{y}=0.1$.

Figure 5 shows the effect of $L_{y}$ on the computed flame front evolution and Fig. 6 the associated propagation rates. For the case with $L_{y}=12.5$ (top panel in Fig. 5) the DL instability is suppressed by thermal conduction and thus the flame flattens after an initial transient period, evolving to steady planar propagation with $S_{T} / S_{L}=1$. As seen in the lower panels of Fig. 5, for larger $L_{y}$, after initial transients the single-cusp structure emerges in a manner similar to that seen in unconfined flames. The effect of the domain width $L_{y}$ on the average propagation rate $\left\langle S_{T} / S_{L}\right\rangle$ is plotted in the inset of Fig. 6 . The value of $\left\langle S_{T} / S_{L}\right\rangle$ is calculated by the slope of the least square fitting in an average $x$-position of the flame vs. time plot (not shown). The average $x$-position of the flame is calculated by weighting the maximum reaction rate over the $y$-domain. Excluding the computations for $L_{y}=200$ (for reasons discussed in the next paragraph), as the domain width increases the propagation speed asymptotes to $\left\langle S_{T} / S_{L}\right\rangle \approx 1.56$.

In the largest lateral domain $\left(L_{y}=200\right)$ we observed sporadic formation of wrinkles on the leading crests of the flame which propagated transversely along the flame surface and disappeared at the trailing cusps. Similar behavior has been observed in computations of freely propagating flames with thermal expansion [36]. These authors showed that while the single-cusp structure is unconditionally stable even for infinitely large $L_{y}$, in practice this solution is never observed; instead, in large domains numerical noise is amplified by the DL instability and the large single-peak structure is contaminated by small nonphysical wrinkles that form and disappear in precisely the manner just described. Consequently, in large lateral domains computations must be performed with high resolution; our relatively coarser resolution $\left(\Delta_{x}=\Delta_{y}=0.1\right)$ for the largest domain $L_{y}=200$ is not quite sufficient to suppress these disturbances. We found that for coarser grid resolutions (not shown) the flame front becomes further contaminated with an increasing number of small-scale wrinkles, thus verifying that the wrinkles we observed were numerical, not physical, in nature. 

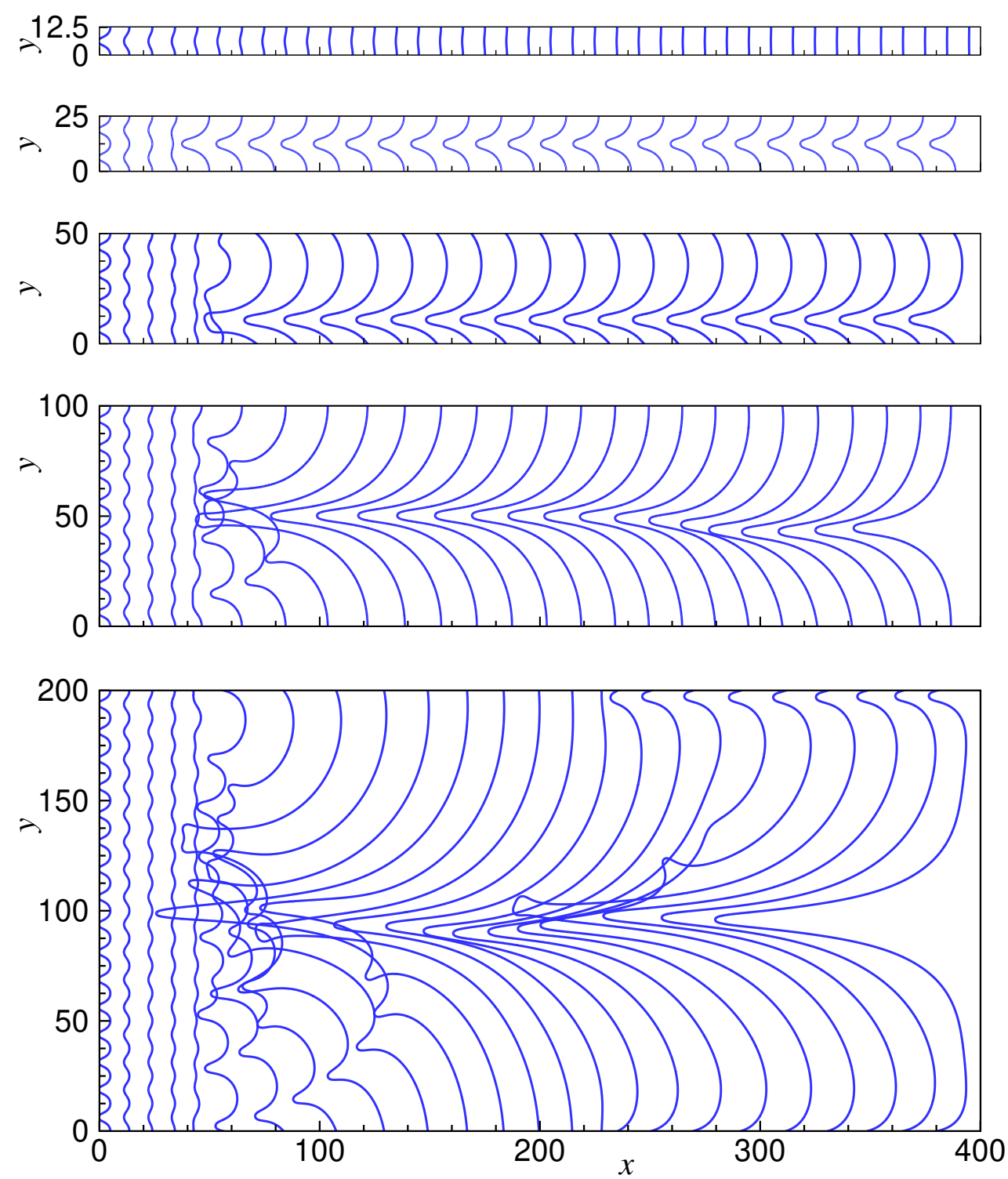

Figure 5: Flame front evolution at time intervals of $t=10$ (except for the first interval at $t=2$ ) characterized by the reaction rate isocontour $\omega=1$. Ignition hot spots at spacings of $d_{s}=12.5$. Lateral domain size (from top to bottom) $L_{y}=12.5,25,50,100$ and 200. The "baseline" case is calculated for $L e=1, \beta=10$, $q=5, G=0$, and $\sigma=0$. Grid resolution $\Delta_{x}=\Delta_{y}=0.05$, except case $L_{y}=200$ where $\Delta_{x}=\Delta_{y}=0.1$. (Animations of the flame evolution for cases with $L_{y}=12.5,25$ and 100 are included in Supplementary materials).

To interpret the effect of instability mechanisms on flame shapes and propagation rates, note that because of the periodic boundary conditions in the $y$-direction, only discrete multiples of wave numbers are allowed, i.e., $k_{1}, k_{2}=2 k_{1}$, and, in general, $k_{n+1}=n k_{1}$. If $k_{n}$ is larger than a neutral-stability wave number $k_{c}=2 \pi / L_{y_{c}}$ separating the unstable and stable ranges then flame wrinkles cannot grow and the flame develops into the planar 


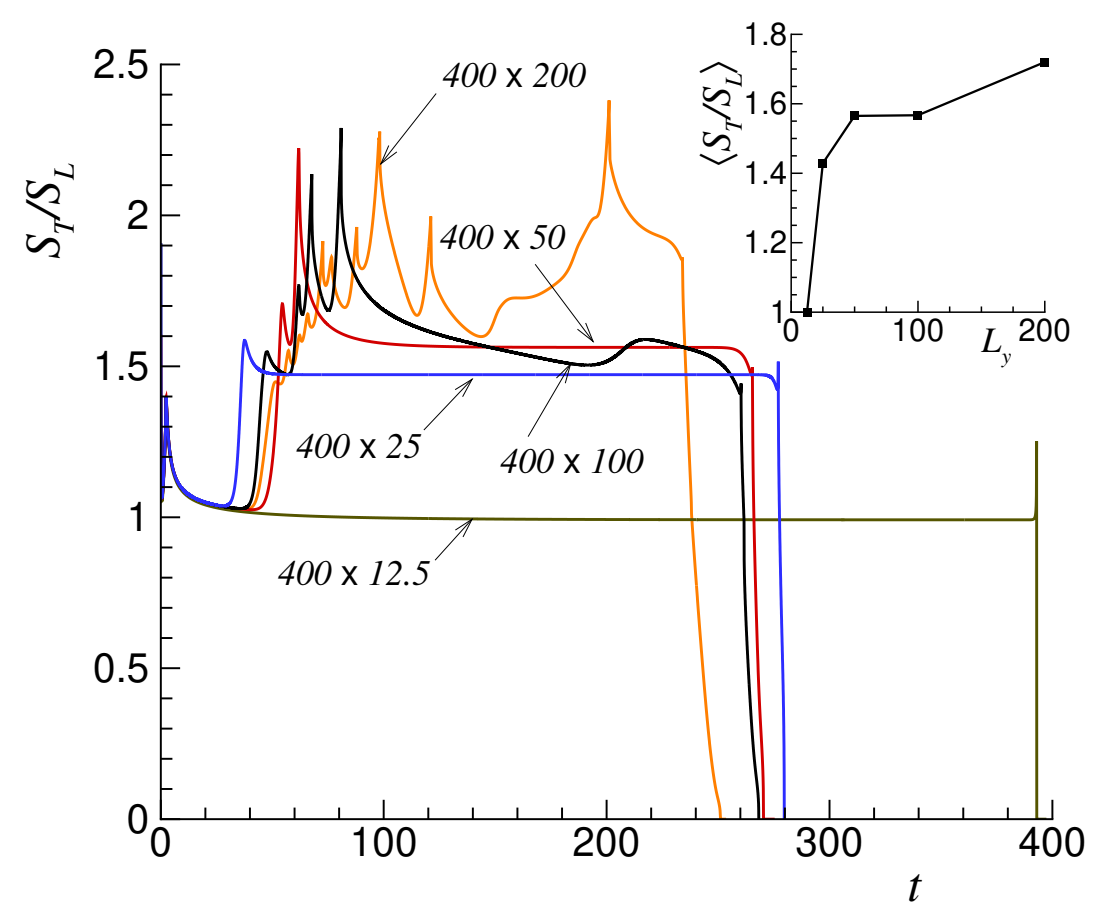

Figure 6: Propagation rate $S_{T} / S_{L}$ vs. time for the cases shown in Fig. 5. The inset shows the variation of the average propagation rate $\left\langle S_{T} / S_{L}\right\rangle$ with $L_{y}$.

solution. This is the case for $L_{y}=12.5$. At some intermediate value of $12.5<L_{y}<25$, the lateral domain size corresponds to a value larger than $L_{y_{c}}$ and the growth of perturbations can be amplified until the wrinkles reach a finite amplitude due to non-linear saturation. The flame shape then evolves to a structure comprised of multiple cusps and eventually a single cusp $[33,36,34,35]$ as we also found in our confined flames. It is also worth noting that the ignition hot spot spacing $d_{s}$ chosen for the calculations in this section is less than the critical wavelength for instability $L_{y_{c}}$ and thus the hot spot configuration does not dictate a particular long-term evolution; the effect of the hot spot spacing is discussed in the following section.

\subsection{Influence of the initial perturbation scale}

To test the influence of the geometry of flame initiation on the subsequent propagation, a series of computations was performed with the "baseline" conditions given in the previous subsection and lateral domain $L_{y}=100$ but varying distances $d_{s}$ between the initial hot spots used for ignition. The resulting flames for $d_{s}=100,50$ and 25 (corresponding to $N=2,3$ and 5, respectively, as compared to $N=9$ for the $L_{y}=100$ case shown in Fig. 5) are shown in Fig. 7. It can be seen that if the distance between the ignition spots is large enough $\left(d_{s} \gtrsim 50\right)$ the number of cusps observed is nearly stable (at least for the longitudinal domain of the computations) and directly related to the configuration of the 
ignition spots. In particular, for $d_{s}=100(N=2)$ a single-cusp mode is obtained whereas for $d_{s}=50(N=3)$ a two-wave mode is observed. For smaller $d_{s}$, the $(N-1)$-wave mode formed initially destabilizes and the flame front inevitably evolves to the single-cusp structure. For the case with $d_{s}=25(N=5)$, the initial flame kernels form a 4-wave mode which propagates nearly steadily for some distance but subsequently destabilizes at $x \approx 120$ $(t \approx 100)$ and forms a two-wave mode, which also propagates steadily for some distance but subsequently destabilizes and finally forms the single-cusp mode. The flame front evolution for the case $d_{s}=12.5(N=9)$ was already shown in Fig. 5 . In that case, the flame evolves directly toward the single-cusp mode after destabilization of the roughly planar shape (an 8-wave mode) at the initial stage. We consistently found that the large amplitude of the curved flame fronts formed as a consequence of the distance between the initial radially growth of the $N$ hot spots for the cases with $d_{s} \gtrsim 50$ is associated with the stability of the $(N-1)$-wave solution with time. The curved flame fronts produce non-linear saturation effects which restrict the growth of DL instabilities [51]. If $d_{s}$ decreases, this saturation does not occur and the $(N-1)$-wave mode destabilizes. When $d_{s}$ is very small, the initial flame shape can be considered as planar and the flame destabilizes as dictated by linear stability analyses.

Figure 8 depicts the calculated propagation rates as a function of time for the aforementioned initial perturbations. In all cases steady propagation of the $(N-1)$-wave mode is observed that only destabilizes for initial hot spot spacings less than $L_{y_{c}}(N=9$ and $N=5)$. In Fig. 8, the instant when the cusps merge coincides with a peak in the propagation rate. A value of $S_{T} / S_{L} \approx 1.6$ is observed during the quasi-steady period.

\subsection{Influence of the viscosity contrast}

In this subsection we examine the effects of the viscosity variation (the driving force of the ST instability) by setting $\sigma=0.7$ and holding fixed all other "baseline" conditions ( $L e=1, \beta=10, q=5$, and $G=0$ ) with $N=3$ initial hot spots used for ignition.

As the actual flame thickness increases when viscosity variation is included, it is convenient to re-scale the computational domain with the ratio $\delta_{f} / \delta_{T}$ given in Table 1 . Specifically, the new computational domain with viscosity variation must be extended from $400 \times 100$ to $1212 \times 303$. This rescaling enables a proper comparison of flame curvatures with the "baseline" case calculated with constant viscosity. (Actually, both cases have the same domain $\left(280 \times 70\right.$ when measured in units of $\left.\delta_{f}\right)$ and the same number of grid points within the flame thickness $\left.\delta_{f}\right)$.

The flame front evolution for the variable-viscosity case is illustrated in Fig. 9. In this case the flames emanating from the three ignition spots propagate faster and the cusps are more elongated compared to the corresponding constant-viscosity case (Fig. 5, middle panel). These elongations produce a significant increase of the total flame length and therefore propagation rate $S_{T} / S_{L}$. The propagation rates (re-scaled with the ratio $\delta_{f} / \delta_{T}$ ) are shown in Fig. 10. The average flame speed for the variable-viscosity case is $S_{T} / S_{L} \approx 2.2$, compared to $S_{T} / S_{L} \approx 1.6$ for the constant-viscosity case. Unlike the constant-viscosity case, however, with variable viscosity the two-cusp structure formed initially cannot be sustained 

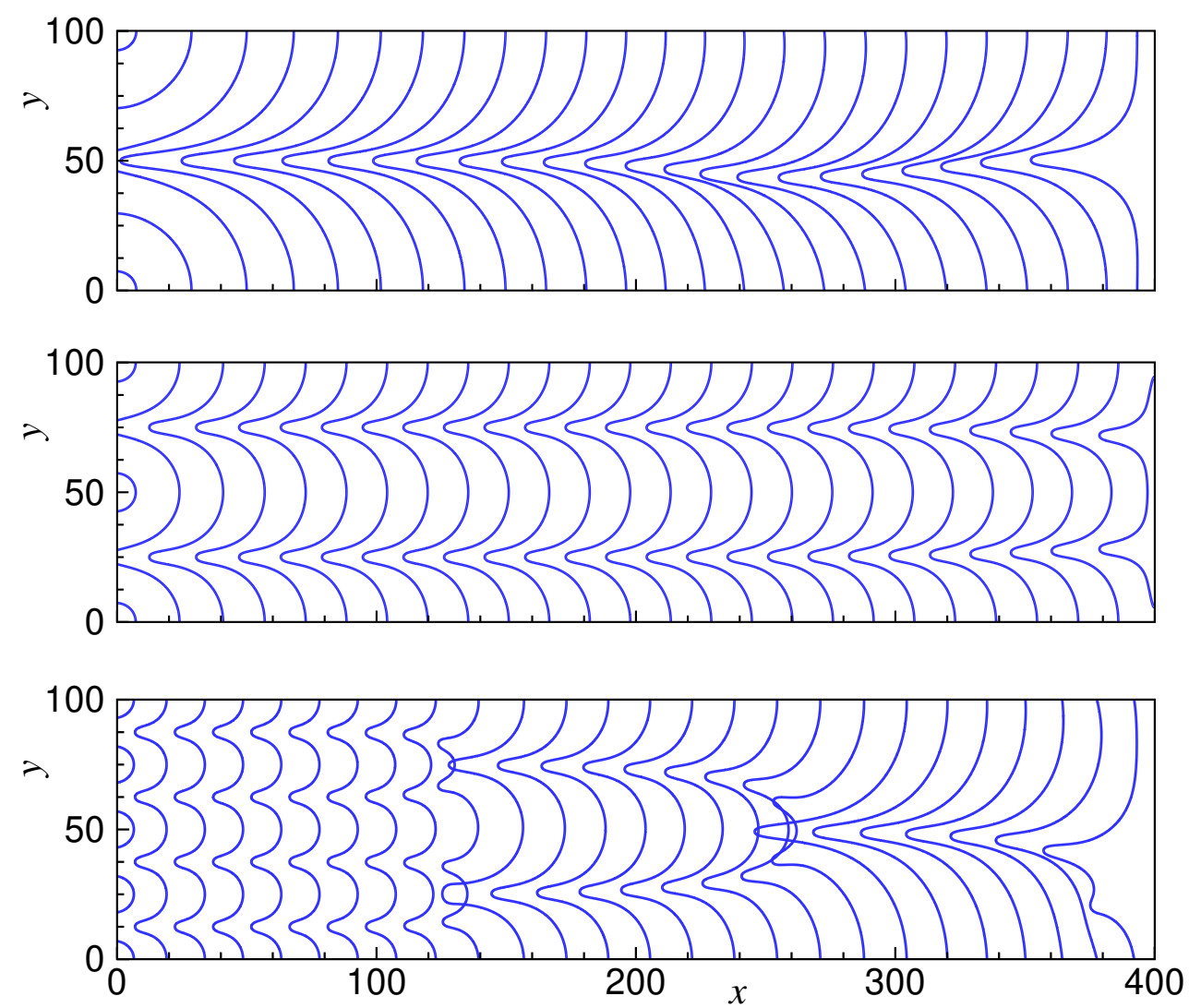

Figure 7: Flame front evolution at time intervals of $t=10$ (except first interval at $t=2$ ) characterized by the reaction rate isocontour $\omega=1$. Initial hot spots for $N=2,3$ and 5 (from top to bottom). The "baseline" case is calculated for $L e=1, \beta=10, q=5, G=0$, and $\sigma=0$. (Animations of the flame evolution for these cases are included in Supplementary materials).

and destabilizes to a 4-wave mode, which later merges back into two cusps which in turn finally merge to form a single cusp. Clearly, the instability mechanisms differ between the two cases because steady flame propagation can not be sustained in the variable-viscosity case. This can be seen in Fig. 10, where the peaks in $S_{T} / S_{L}$ correspond to cell merging and the drops in $S_{T} / S_{L}$ correspond to the disappearance of cusps. The evolution of the total flame length normalized with lateral domain size, $l_{f} / L_{y}$ is also shown in Fig. 10 (dashed curves). For both constant-viscosity and variable-viscosity cases there is practically no difference between $S_{T} / S_{L}$ and $l_{f} / L_{y}$ and in fact there is so little difference for the constant-viscosity case that the two are indistinguishable. This demonstrates that for both cases the propagation speeds are associated with the total flame length rather than variations in the local laminar burning velocity $S_{L}$.

To better explain the process, we show in Fig. 11 wrinkled flame shapes (scaled with the ratio $\delta_{f} / \delta_{T}$ ) extracted at different instants marked with symbols in Fig. 10 superimposed on the streamline patterns. Flow accelerates downstream as it approaches the flame crests 


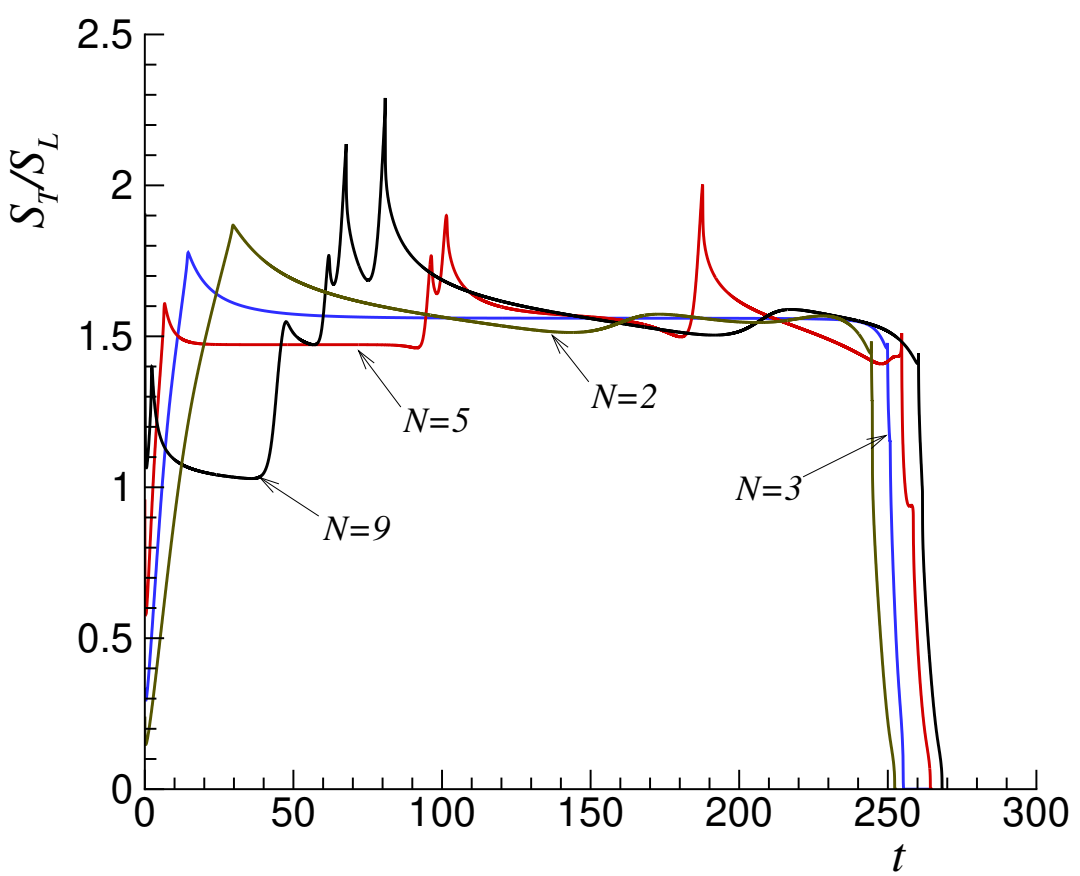

Figure 8: Propagation rate $S_{T} / S_{L}$ vs. time for the different initial perturbations shown in Fig. $7(N=2,3$ and 5$)$ and in Fig. $5\left(N=9\right.$ for the $L_{y}=100$ case).

(the part of the flame that is convex toward the fresh mixture) and is rapidly diverted and concentrated into the troughs (the part of the flame that is concave toward the fresh mixture). Initially, both cases shown exhibit a two-wave mode of propagation but the cusps are significantly deeper in the variable-viscosity case. The gas expansion, combined with the effects of confinement, produces large pressure gradients that accelerates the burnt gases to the left end of the domain. When the viscosity in the burnt region increases (3.5 times the viscosity of the fresh mixture for $\sigma=0.7$ ) it produces a larger increment of pressure gradient which further elongates the cusps in comparison with the constant viscosity case, increasing the total flame length. If the flame front is initially wrinkled, i.e., due to initial two-wave mode of perturbation, the convective flow concentrates into the cusps (flame troughs) and elongates the flame, increasing the flame area and thus propagation velocity.

Pressure profiles in the $x$ direction, i.e., the mean propagation direction, taken along the centerline of the cell in the $y$ direction at several instants in time, are shown in Fig. 12. Ahead of the flame there is practically no gas motion (recall that the flame is propagating towards the closed end of the cell) and thus no pressure gradient whereas behind the flame in the burned cases the pressure gradient is nearly constant. Variations of pressure along the lateral $(y)$ coordinate (not shown) were found to be negligible because the flame propagates essentially in the $x$ direction. With variable viscosity, the pressure increases are an order of 


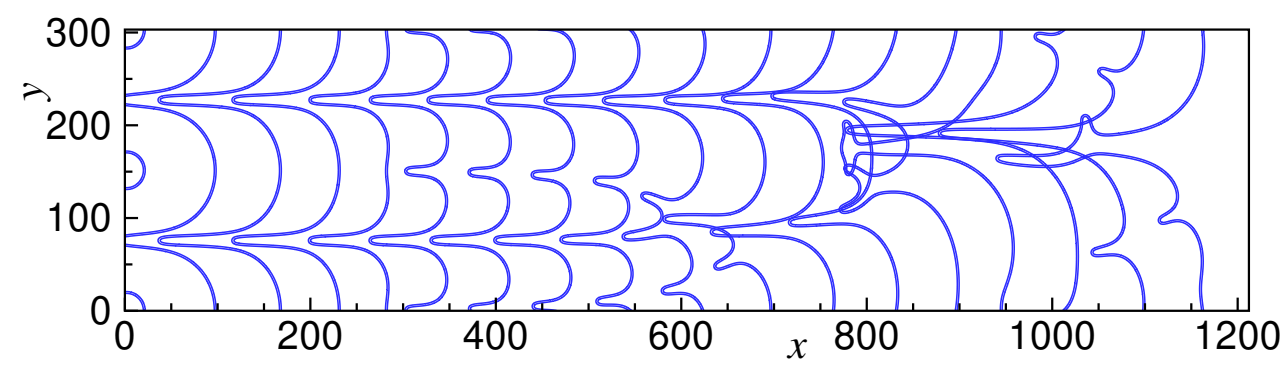

Figure 9: Flame front evolution at time intervals of $t=30$ (except first interval at $t=6$ ) characterized by the reaction rate isocontour $\omega=0.2$. Calculated for $L e=1, \beta=10, q=5, G=0$, and $\sigma=0.7$. (Animation of the flame evolution for this case is included in Supplementary materials).

magnitude larger than with constant viscosity. This is expected considering that these pressure increases are of the order $\Delta p \sim \mu U L$ and that variable viscosity case causes viscosity, propagation rate and length scale increases (compared to baseline constant viscosity case) by factors of about 3.5, 1.5 and 3 , respectively.

The $x$-component of the gas velocity $\left(U_{x}\right)$ induced by pressure gradient at instant $t /\left(\delta_{f} / \delta_{T}\right)=$ 76 is shown in Fig. 13. The velocity is measured along the line $y /\left(\delta_{f} / \delta_{T}\right)=17.5$ (in coincidence with a cusp, at least during the initial evolution) and at the middle section $y /\left(\delta_{f} / \delta_{T}\right)=35$. In both sections $U_{y}$ is almost negligible and the magnitude of $U_{x}$ corresponds approximately to the total velocity magnitude. Fig. 13 confirms the larger outlet gas velocity at the left (open) end of the cell for the variable-viscosity case (due to the larger $S_{T} / S_{L}$ ) and the larger convective flow concentrated in the cusps. If we integrate the mass conservation equation (15) across the plates in a reference frame moving with the flame at $S_{T} / S_{L}$ and make use of the boundary conditions (22)-(24), we obtain $U_{x}(x=0)=-\left(S_{T} / S_{L}\right) q$. This indicates that the minimum velocity of the outlet gas is given by the planar flame condition, with $U_{x}(x=0)=-q$. If the flame is wrinkled or more elongated, as is the case with variable viscosity, more flame surface area is created and the outlet gas velocity at the open end increases. The larger (negative) velocities appear at the cusps and for variable viscosity. As can be seen in the lower plot of Fig. 13, there is a small acceleration of the fresh gases ahead of the flame (at the flame crests) in the direction of the flame propagation caused by thermal expansion. As previously mentioned the fresh gas flow is quickly diverted into the opposite direction toward the flame troughs, inducing straining on the elongated flame front. The additional straining found on the flame surface for the variable-viscosity case promotes the appearance of a new instability at the hydrodynamic scale that destabilizes the wrinkled flame. The effect of this mechanism is clearly seen in the lower plots of Fig. 11 where the leading part of the cell during the initial two-wave mode at $t /\left(\delta_{f} / \delta_{T}\right)=21$ is split into two cells, forming a four-wave mode at $t /\left(\delta_{f} / \delta_{T}\right)=49$ that resembles the classical finger-like structures reported in viscous fluids driven in porous medium [28], which strongly indicates that this mechanism is associated with the ST instability.

Calculations neglecting the transverse confinement [33, 34, 35, 36, 37], that is, for purely 


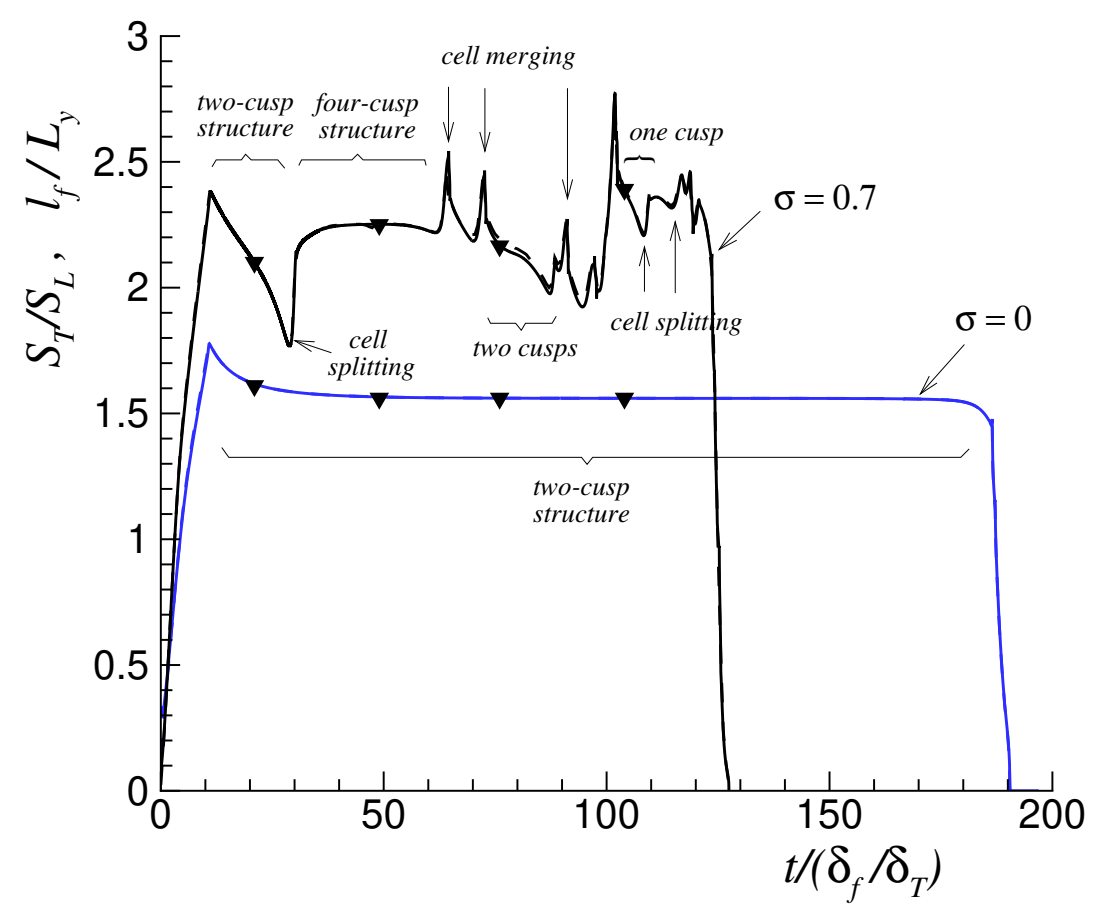

Figure 10: Time evolution of the propagation rate for the cases with $\sigma=0.7$ and $\sigma=0$. The symbols correspond with the flame structures showed in Fig. 11. Dashed curves indicates the total flame length normalized by the lateral size $L_{y}$. Calculated for $L e=1, \beta=10, q=5$, and $G=0$ in a domain of $400 \times 100$ $(\sigma=0)$ and $1212 \times 303(\sigma=0.7)$.

2D freely propagating flames without confinement, predict a propagation speed of $S_{T} / S_{L} \approx$ 1.2. This speed increases when the transverse confinement is included in Hele-Shaw cells, through expansion-induced straining effects, to $S_{T} / S_{L} \approx 1.4$ if $a=15$ [32] and to $S_{T} / S_{L} \approx$ 2.2 in the limit of large viscosity effects $(a \rightarrow 0)$ proposed in the present work. This value compares reasonably well with those found in experiments in lean $\mathrm{CH}_{4}$-air mixtures $\left(S_{T} / S_{L} \approx 2.5\right)$ [39], for which the Lewis number of the mixture is not far from unity and the thermal expansion coefficient is close to that employed in our computations $(q=5)$.

\subsection{Influence of thermal expansion}

Figure 14 shows propagation rates $S_{T} / S_{L}$ for varying thermal expansion coefficient $q$ (thus varying DL effect) with all other conditions fixed ( $L e=1, \beta=10, G=0, \sigma=0.7$, domain $200 \times 100\left(46 \times 23\right.$ in units of $\delta_{f}$ and $N=3$ initial hot spots. (The $N=3$ hot spots were employed to generate a steady two-wave mode of propagation, as discussed in previous subsections.) Fig. 14 shows that the flame propagates quasi-steadily after some initial acceleration, with larger values of $S_{T} / S_{L}$ for increasing $q$. The dependence of the quasi-steady $S_{T} / S_{L}$ with $q$ is nearly linear as indicated in the inset of this figure. We also display in Fig. 14 (dashed curve) the total flame length normalized by the lateral domain 

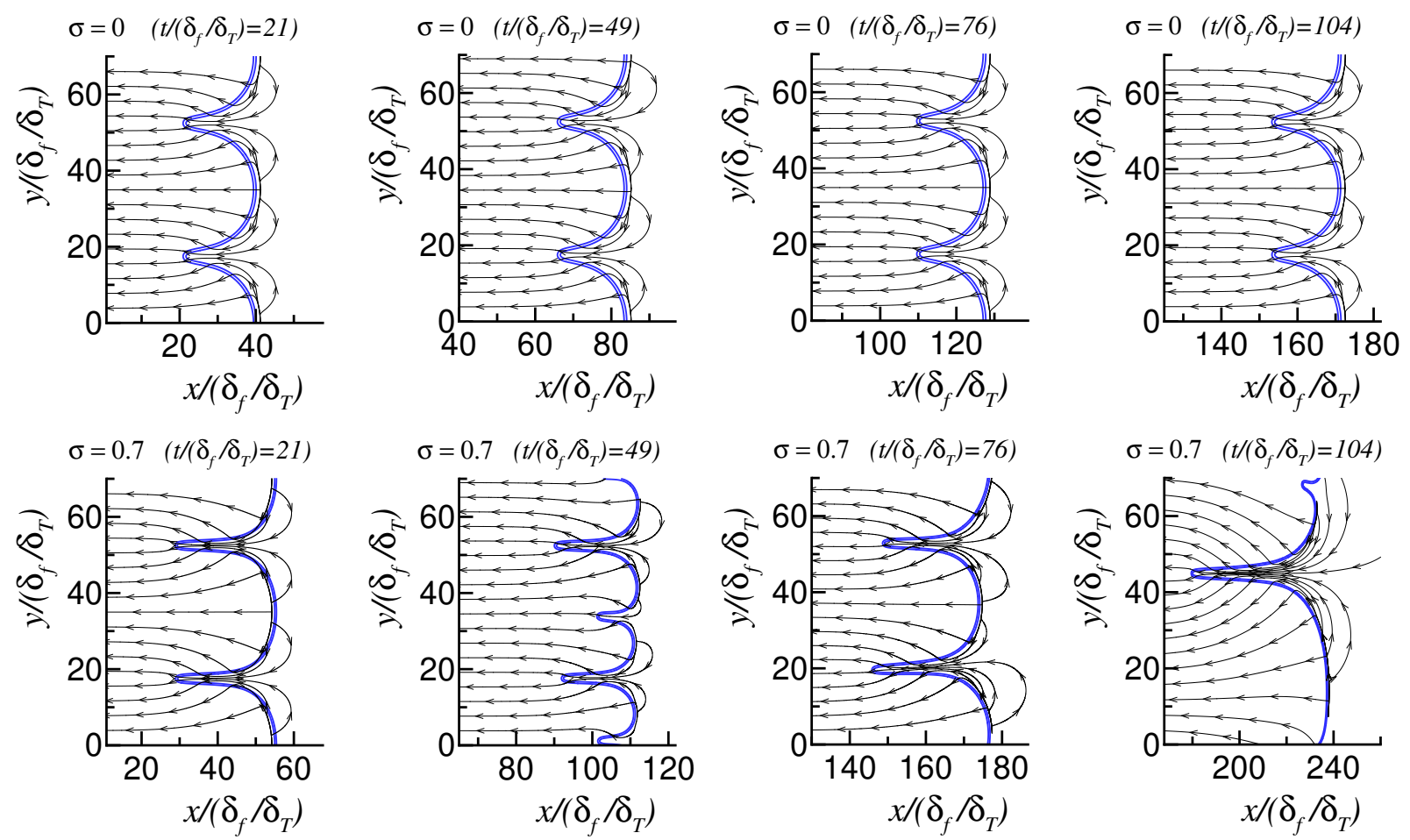

Figure 11: Illustration of the wrinkled flame front represented by the reaction rate isocontour $(\omega=0.2)$ and the flow field by the streamline pattern, at different instants for $\sigma=0$ (top) and $\sigma=0.7$ (bottom). Extracted from Figs. 7 and 9.

size, $l_{f} / L_{y}$, although the correspondence between propagation rate and flame length is so close that the dashed curve is almost indistinguishable from the solid curve. This indicates that (as was demonstrated for the effect of viscosity variation as described in the previous subsection) the increase of $S_{T} / S_{L}$ due to thermal expansion is associated with the increase of flame surface rather than local modifications to $S_{L}$.

Figure 15 shows flow patterns for the steady two-wave mode of propagation at the instants marked with symbols in Fig. 14. It can be seen that thermal expansion induces an increase in the straining which elongates the troughs and therefore increases the flame length. Larger values of $q$ sharpen the cusps whereas smaller values corrugate the flame surface only slightly. In particular, it is interesting to note the case with $q=5$. It shows a steady two-wave mode of propagation when the domain width is $L_{y}=100$ but not when $L_{y}=303$ (see Fig. 9), indicating that the critical wavelength of instability $L_{y_{c}}$ is reduced when the viscosity variation is included.

\subsection{Influence of buoyancy}

Figure 16 shows propagation rates $S_{T} / S_{L}$ for varying values of the buoyancy parameter $G$ with all other conditions fixed $(L e=1, \beta=10, q=5, G=0, \sigma=0.7$, domain $200 \times 100\left(46 \times 23\right.$ in units of $\left.\delta_{f}\right)$ and $N=3$ initial hot spots. As expected, for $G>0$ 

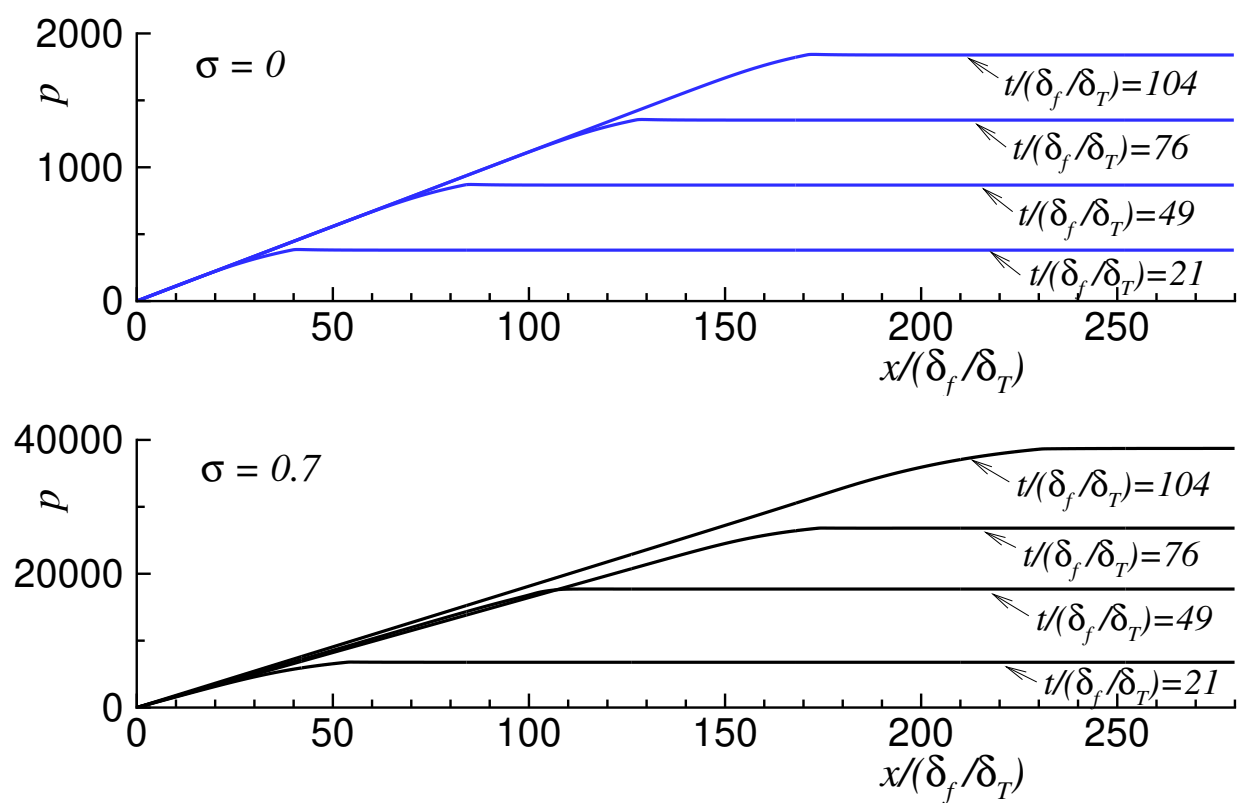

Figure 12: Pressure variations along the plates measured at middle sections and at different instants. Upper plot: constant-viscosity case in a domain $400 \times 100$; lower plot: variable viscosity in a domain $1212 \times 303$. Calculated for $L e=1, \beta=10, q=5$, and $G=0$.

(downward propagation, where RT effects are stabilizing) $S_{T} / S_{L}$ decreases due to a decrease in amplitude of the flame wrinkles. In particular, above a critical value of the buoyancy parameter $G \approx 12$ the stabilizing influence of the RT mechanism dominates the destabilizing influences of the DL and ST mechanisms and the planar solution emerges, see Fig. 17. For $G<0$ (upward propagation) the troughs elongate, increasing the flame surface area and thus the propagation rates. The inset of Fig. 16 shows that the dependence of the propagation speed on $G$ is approximately linear except when the flame is nearly planar.

\subsection{Influence of differential diffusion}

Figure 18 shows time-dependent propagation rates $S_{T} / S_{L}$ for varying values of the Lewis number $L e$ with all other conditions fixed $(\beta=10, q=5, G=0, \sigma=0.7, G=0$, domain $200 \times 100\left(46 \times 23\right.$ in units of $\delta_{f}$ and $N=3$ initial hot spots. $)$ The time-averaged propagation rates are shown in the inset of Fig. 18. To facilitate comparisons with the experiments in [38], a smaller value of the thermal expansion parameter $(q=3)$ was employed than that used in previous calculations. Steady cell formation and propagation is found for $L e>0.8$. For $L e=0.7$ we find small lateral movement of cells in a quasi-steady manner. For $L e \geq 0.7$, after some initial transients the $S_{T} / S_{L}$ converges to a quasi-steady value. For $L e \leq 0.7$ chaotic cell splitting and merging, qualitatively similar to that found in the experiments [38], was observed; in this case the propagation is inherently unsteady, thus we report an average propagation speed, $\left\langle S_{T} / S_{L}\right\rangle$, as defined above. The diffusive-thermal instability causes $\left\langle S_{T} / S_{L}\right\rangle$ to attain very large values for low $L e$ whereas for $L e \geq 2$ DT effects are 

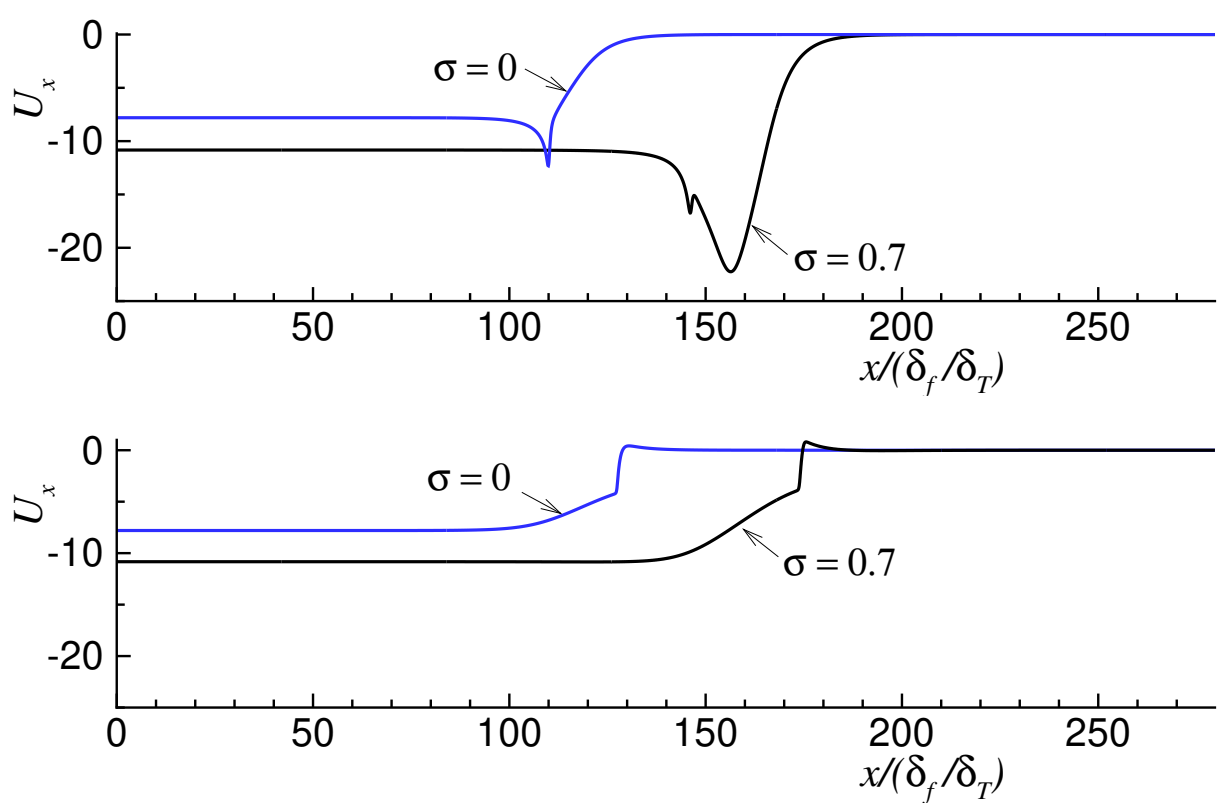

Figure 13: Gas velocity, $U_{x}$, along the plates measured in the section coinciding with the position of a cusp at $y /\left(\delta_{f} / \delta_{T}\right)=17.5$ (upper plot) and in the middle section at $y /\left(\delta_{f} / \delta_{T}\right)=35$ (lower plot) for instant $t /\left(\delta_{f} / \delta_{T}\right)=76$.

sufficiently stabilizing that the flames become nearly flat and $\left\langle S_{T} / S_{L}\right\rangle \rightarrow 1$.

Figure 19 shows reaction rate contours along with flow patterns for varying Le. For $L e<1$ ( Le $>1$ ) the reaction rate increases (decreases) on the convex part of the flame with respect to the unburnt side. For low Le the propagation rate is dependent not only on flame length but also to the local enhancement of the reaction rate, and thus there are substantial differences between the values of $S_{T} / S_{L}$ (solid curves) and $l_{f} / L_{y}$ (dashed curves) in Fig. 18 that were not present for $L e=1$. As a consequence, for $L e \leq 0.7$ the flame speed enhancement is far more than that due to the flame length enhancement.

The cell pattern seen in the bottom right-hand corner of Fig. 19 shows a striking similarity to a portion of the experimental image shown in Fig. 3 for a lean $\mathrm{H}_{2}-\mathrm{O}_{2}-\mathrm{N}_{2}$ mixture (for which $L e \approx 0.34$ and $q \approx 3.1$ ). In particular, note that for low Le the large-scale features exhibit an angular or "sawtooth" structure. It should be noted, however, that the calculated average propagation speed $\left(\left\langle S_{T} / S_{L}\right\rangle \approx 7\right)$ is about half that observed in the experiments $\left(\left\langle S_{T} / S_{L}\right\rangle \approx 15\right)$. For larger $L e$, the large-scale structure is cusped rather than angular, which is also consistent with the experimental observation (Fig. 2). Consequently, our model is able to capture an important aspect of interactions of the diffusive-thermal (DT) and hydrodynamic (DL and ST) instabilities observed in experiments. 


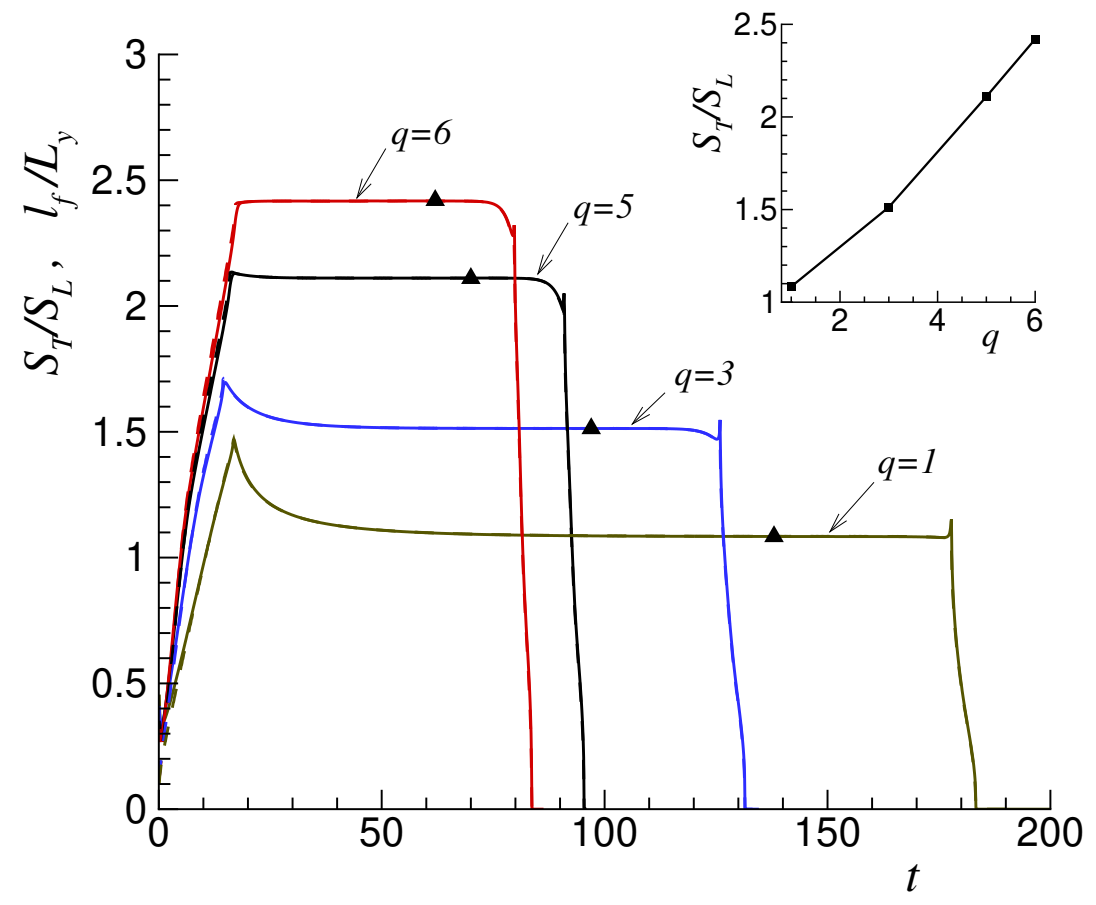

Figure 14: Propagation rates $S_{T} / S_{L}$ as a function of time for different values of the thermal expansion coefficient $q$ in a domain of $200 \times 100$. Dashed curves indicate the total flame length normalized by the lateral size $L_{y}$. The symbols correspond with the flames shown in Fig. 15. Calculated for $L e=1, \beta=10$, $G=0$, and $\sigma=0.7$. The inset shows the effect of $q$ on the quasi-steady value of $S_{T} / S_{L}$.

\section{Conclusions}

The limit $a \rightarrow 0$, where $a=h / \delta_{T}$ is the ratio of the plate separation $h$ to the thermal flame thickness $\delta_{T}$, is proposed for investigating quasi-isobaric flame propagation between two narrowly-spaced adiabatic parallel plates, a configuration known as a Hele-Shaw cell. The resulting quasi-2D formulation obtained in this limit, which employs one-step chemistry with constant heat capacity but variable transport coefficients, is equivalent to solving the energy and species equations with quantities averaged across the cell gap and with the flow field governed by Darcy's Law. The advantage of this approach is that the computational cost in large domains is greatly reduced compared with fully 3D simulations.

The present work considered flames propagating from an open end towards a closed end with periodic boundary conditions in the lateral dimension. The effect of the lateral extension and the initial perturbation scale was first investigated for an equidiffusional $(L e=$ 1) "baseline" case. Large lateral domains of integration are very sensitive to the level of numerical noise so computations must be performed on a very fine mesh, typically with at least 16 points within the actual flame thickness. The flame is initiated at the (left) open end with a series of $N$ hot spots uniformly distributed which subsequently form a $(N-1)$-wave 

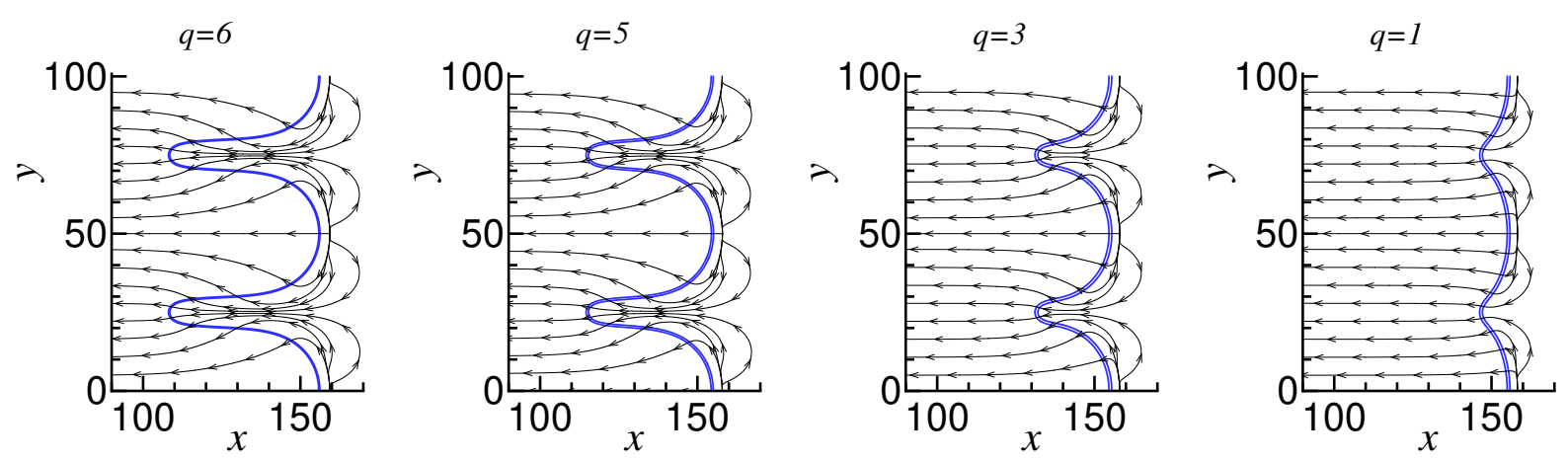

Figure 15: Illustration of the steady flame front represented by the reaction rate isocontour $(\omega=0.5)$ and the flow field by the streamline pattern at the different values of the thermal expansion coefficient $q$ marked with symbols in Fig. 14. (Animations of the flame evolution for the cases $q=5$ and $q=1$ are included in Supplementary materials).

mode of propagation during the first stage of the flame evolution. If the distance between the spots is sufficiently large, the $(N-1)$-wave mode is stable and the structure propagates steadily until the flame reaches the closed end. Otherwise, the $(N-1)$ waves destabilize, showing cell merging that eventually evolves toward a flame structure with a single cusp, independently of the lateral domain size. For lateral extents below the critical wavelength of instability $L_{y_{c}}$ (as it is the case for $L_{y}=12.5$ ) the flame evolves to the planar solution.

In the second part of the work, we investigated the effects of instability mechanisms associated with thermal expansion, buoyancy, viscosity contrast and differential diffusion on the flame dynamics and propagation rates. Because of the confinement, the viscosity change across the flame induces an additional wrinkling mechanism beyond that caused by thermal expansion which further elongates the flame surface and increases the flame propagation rate. In particular, the calculated propagation rates $\left(S_{T} / S_{L} \approx 2.2\right)$ when $L e=1$ show good agreement with the experimental values reported in lean methane-air mixtures [39]. Buoyancy effects increase/decrease the total flame length (and therefore the propagation rates) in linear proportion with the buoyancy parameter $G$ for flames spreading in the opposite/same direction, as anticipated by Joulin and Sivashinsky [31]. A critical constant, $G=12$, above which the stabilizing RT mechanism can suppress DL and ST instabilities, is identified in the computations. An estimated order of magnitude of the parameter $a$ for this critical buoyancy value in lean $\mathrm{CH}_{4}$-air experiments (for which $\mathcal{D}_{T_{u}}=2.2 \cdot 10^{-5} \mathrm{~m}^{2} / \mathrm{s}$ ) gives $a=\left(12 \operatorname{Pr} G S_{L}^{3} / g \mathcal{D}_{T_{u}}\right)^{1 / 2} \approx 170$, indicating that planar flame fronts could be observed, in principle, for downward propagating flames. For the lean $\mathrm{H}_{2}-\mathrm{O}_{2}-\mathrm{N}_{2}$ mixture of Fig. 4 (for which $\mathcal{D}_{T_{u}}=3.2 \cdot 10^{-5} \mathrm{~m}^{2} / \mathrm{s}$ ) buoyancy stabilizes the hydrodynamic effects at $a \approx 23$, demonstrating that the large-scale structure of this image should be relatively flat flatter due to the RT effect, which is consistent with Fig. 4.

The most striking results were found for simulations with small Lewis numbers, where DT instabilities promote the appearance of small cells embedded into large-scale hydrodynamic structures that typically show an angular shape. The flame shapes obtained during the 


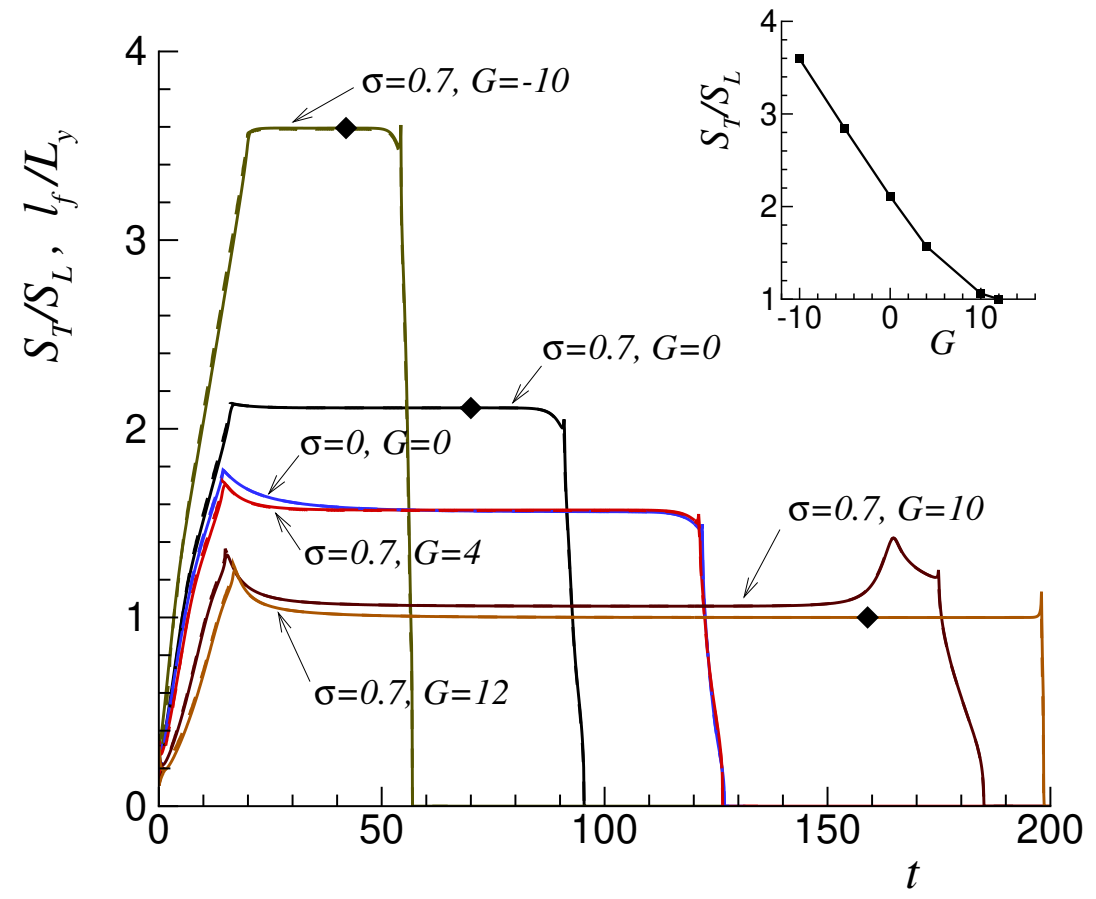

Figure 16: Propagation rates $S_{T} / S_{L}$ as a function of time for different values of the buoyancy paramenter $G$ in a domain of $200 \times 100$. Dashed curves indicate the total flame length normalized by the lateral size $L_{y}$. The symbols correspond with the flames shown in Fig. 17. Calculated for $L e=1, \beta=10, q=5$ and $\sigma=0.7$. The inset shows the effect of $G$ on the quasi-steady value of $S_{T} / S_{L}$.

simulations for $L e=0.3$ are in good agreement with those found in lean $\mathrm{H}_{2}-\mathrm{O}_{2}-\mathrm{N}_{2}$ mixtures [38], but calculated propagation rates $\left(S_{T} / S_{L} \approx 7\right)$ are half than those of the experiments $\left(S_{T} / S_{L} \approx 15\right)$. For finite cell gaps, the DT instability can also induce flame curvature in the third dimension which would further increase the flame surface and therefore the propagation rates, i.e., curved non-symmetric flames arise in narrow planar channels due to small-scale DT instabilities [11]. This could explain, in principle, the discrepancies found between our computations and the experiments in terms of the values of the propagation rates for lean $\mathrm{H}_{2}-\mathrm{O}_{2}-\mathrm{N}_{2}$ mixtures. Experiments with leaner mixtures than that shown in Fig. 3 may decrease the value of the paramenter a reducing the curvature of the flame in the third dimension and the propagation speed to values similar to those calculated in the present work. A potential additional source of discrepancy is that associated with the Soret diffusion, which is of relevance for hydrogen mixtures but was not included in our simulations. Its effect, being similar to having a smaller effective Lewis number, would increase the calculated propagation speeds. Inclusion of heat losses to the walls may also influence on the propagation rates, but in the opposite direction. For example, if small heat losses of the order $a$ are included in the formulation by changing the corresponding boundary conditions (10) for the temperature to $\mu \partial \theta / \partial z+a^{2} b \theta=0$ in the upper plate 

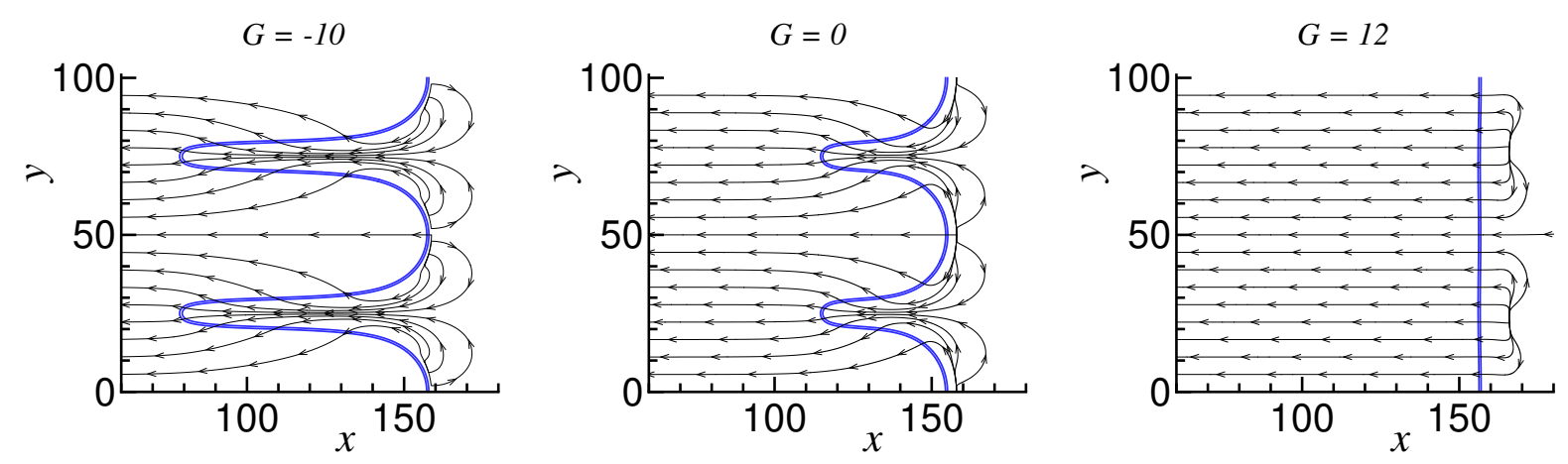

Figure 17: Illustration of the flame front represented by the reaction rate isocontour $(\omega=0.5)$ and the flow field by the streamline pattern at the different values of the buoyancy parameter $G$ marked with symbols in Fig. 16. (Animations of the flame evolution for the cases $G=-10$ and $G=12$ are included in Supplementary materials).

and $\mu \partial \theta / \partial z-a^{2} b \theta=0$ in the lower plate, with $b=\left(\lambda_{\mathrm{w}}^{\prime} / \lambda^{\prime}\right)\left(\delta_{T} / h_{\mathrm{w}}\right)\left(\delta_{T} / h\right)$ being the heatloss parameter, the integrated first order term in the left hand side of Eq. (17) will remain with a negative value $-2 b \theta_{0}$. This value enters to stabilize the zeroth order solution and, consequently, to reduce the calculated propagation speeds. When $b \rightarrow 0$ we recover the adiabatic solution, when $b \rightarrow 1 / a$ we have isothermal walls and the solution corresponds to $\theta_{0}=Y_{0}-1=0$, and in the distinguished limit $b \rightarrow 1$ small heat losses can be included in the analysis (here $\lambda_{\mathrm{w}}^{\prime}$ and $h_{\mathrm{w}}$ are the thermal conductivity and thickness of the solid walls, respectively.)

Despite the fact that the parameter $a$ has been considered very small and that heat losses were not taken into account, the present formulation shows reasonably good agreement with the experimental observations, both qualitatively and in many cases quantitatively. A more detailed quantification of the range of validity of this quasi-2D limit with careful 3D simulations will be performed in the future.

\section{Acknowledgments}

This research was supported by the project ENE2015-65852-C2-2-R and has received funding from the European Union's Horizon 2020 Programme (2014-2020) and from Brazilian Ministry of Science, Technology and Innovation through Rede Nacional de Pesquisa (RNP) under the HPC4E Project (www.hpc4e.eu), grant agreement number 689772. The USC work was supported by the U.S. National Science Foundation, grant number CBET1236892. DFG gratefully acknowledges the invitation of PDR to visit USC during winter 2013 and would also like to thank J. Gross at USC for his hospitality during the visit. DFG also acknowledges helpful discussions with Dr. Rosa E. Padilla.

\section{References}

[1] A. C. Fernández-Pello, Micropower generation using combustion: issues and approaches, Proc. Combust. Inst. 29 (2002) 883-899. 


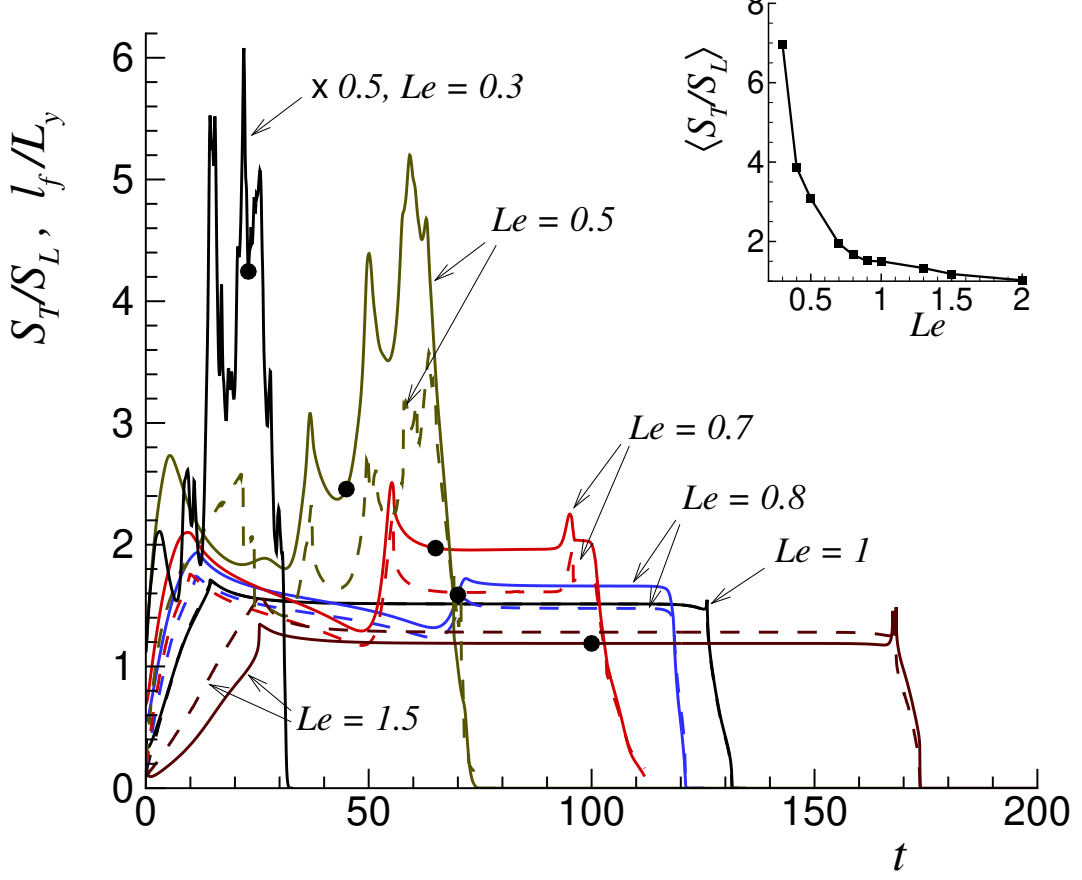

Figure 18: Propagation rates $S_{T} / S_{L}$ as a function of time for different values of Le. Dashed curves indicate the total flame length normalized by the lateral size $L_{y}$. The symbols correspond to flames shown in Fig. 19 . Calculated for $\beta=10, q=3, G=0$, and $\sigma=0.7$ in a domain of $200 \times 100$. The inset shows the effect of Le on the mean propagation rate $\left\langle S_{T} / S_{L}\right\rangle$.

[2] D. Dunn-Rankin, E. M. Leal, D. C. Walther, Personal power systems, Prog. Energ. Combust. Sci. 31 (2005) 422-465.

[3] K. Maruta, Micro and mesoscale combustion, Proc. Combust. Inst. 32 (2011) 125-150.

[4] Y. Ju, K. Maruta, Microscale combustion: Technology development and fundamental research, Prog. Energ. Combust. Sci. 37 (2011) 669-715.

[5] D. C. Walther, J. Ahn, Advances and challenges in the development of power-generation systems at small scales, Prog. Energ. Combust. Sci. 37 (2011) 583-610.

[6] N. S. Kaisare, D. G. Vlachos, A review on microcombustion: Fundamentals, devices and applications, Prog. Energ. Combust. Sci. 38 (2012) 321-359.

[7] C. M. Miesse, R. I. Masel, C. D. Jensen, M. A. Shannon, M. Short, Submillimeter-scale combustion, AIChE J. 50 (2004) 3206-3214.

[8] S. Y. Jejurkar, D. P. Mishra, A review of recent patents in micro-combustion and applications, Recent Patents on Engineering 3 (3) (2009) 194-209.

[9] J. Daou, M. Matalon, Influence of conductive heat-losses on the propagation of premixed flames in channels, Combust. Flame 128 (2002) 321-339.

[10] V. N. Kurdyumov, E. Fernández-Tarrazo, Lewis number effect on the propagation of premixed laminar flames in narrow open ducts, Combust. Flame 128 (2002) 382-394.

[11] V. N. Kurdyumov, Lewis number effect on the propagation of premixed flames in narrow adiabatic channels: Symmetric and non-symmetric flames and their linear stability analysis, Combust. Flame 158 (2011) 1307-1317. 

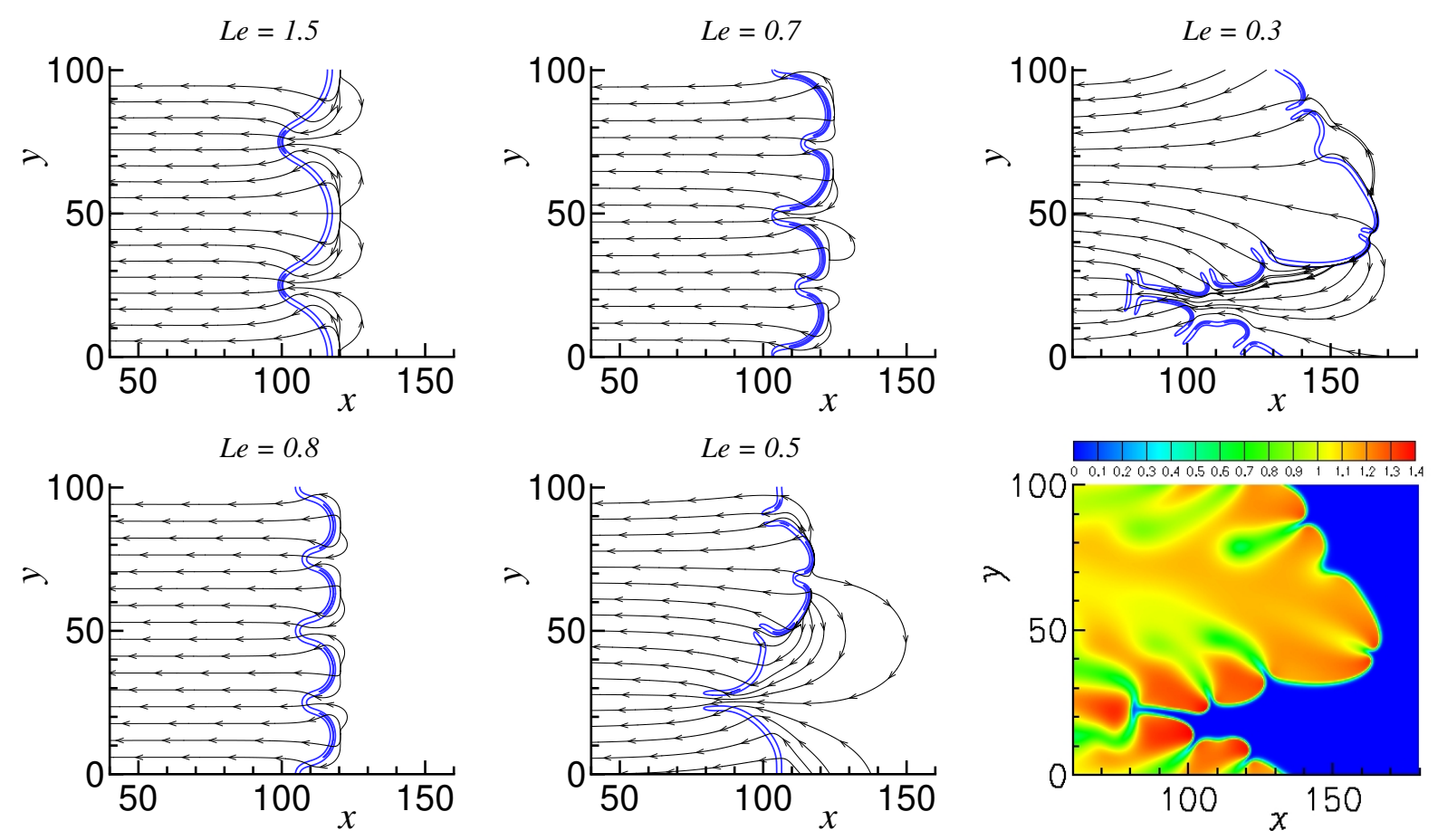

Figure 19: Illustration of the flame fronts indicated by the reaction rate isocontour $(\omega=0.2,1$ for $L e=1.5$, $0.8,0.7 ; \omega=0.2,2$ for $L e=0.5$; and $\omega=0.2,5$ for $L e=0.3$ ) along with the flow field streamline patterns for different values of Le. Calculated for $\beta=10, q=3, \sigma=0.7$, and $G=0$. The plot in the bottom right-hand corner shows maps of temperature (colour online) for the case $L e=0.3$. (Animations of the flame evolution for the cases with $L e=1.5, L e=0.8$ and $L e=0.3$ are included in Supplementary materials).

[12] V. N. Kurdyumov, C. Jiménez, Propagation of symmetric and non-symmetric premixed flames in narrow channels: influence of conductive heat-losses, Combust. Flame 161 (2014) 927-936.

[13] D. Fernández-Galisteo, C. Jiménez, M. Sánchez-Sanz, V. N. Kurdyumov, The differential diffusion effect of the intermediate species on the stability of premixed flames propagating in microchannels, Combust. Theory Modelling 18 (4-5) (2014) 582-605.

[14] B. Bai, Z. Chen, H. Zhang, S. Chen, Flame propagation in a tube with wall quenching of radicals, Combust. Flame 160 (2013) 2810-2819.

[15] M. Short, D. A. Kessler, Asymptotic and numerical study of variable-density premixed flame propagation in a narrow channel, J. Fluid Mech. 638 (2009) 305-337.

[16] V. N. Kurdyumov, M. Matalon, Flame acceleration in long narrow open channels, Proc. Combust. Inst. 34 (2013) 865-872.

[17] P. Pearce, J. Daou, Taylor dispersion and thermal expansion effects on flame propagation in a narrow channel, J. Fluid Mech. 754 (2014) 161-183.

[18] V. N. Kurdyumov, M. Matalon, Self-accelerating flames in long narrow open channels, Proc. Combust. Inst. 35 (2015) 921-928.

[19] S. T. Lee, C. H. Tsai, Numerical investigation of steady laminar flame propagation in a circular tube, Combust. Flame 99 (1994) 484-490.

[20] N. I. Kim, K. Maruta, A numerical study on propagation of premixed flames in small tubes, Combust. Flame 146 (2006) 283-301.

[21] G. Pizza, C. E. Frouzakis, J. Mantzaras, A. G. Tomboulides, K. Boulouchos, Dynamics of premixed hydrogen/air flames in microchannels, Combust. Flame 152 (2008) 433-450. 
[22] C. Jiménez, D. Fernández-Galisteo, V. Kurdyumov, DNS study of the propagation and flashback conditions of lean hydrogen-air flames in narrow channels: symmetric and non-symmetric solutions, Int. J. Hydrogen Energy 40 (2015) 12541-12549.

[23] H. J. S. Hele-Shaw, The flow of water, Nature 58 (1898) 34-36.

[24] A. Vasil'ev, From the Hele-Shaw experiment to integrable systems: A historical overview, Compl. Anal. Oper. Theory 3 (2) (2009) 551-585.

[25] G. Darrieus, Propagation d'un front de flamme, unpublished work presented at La Technique Moderne, Paris (1938).

[26] L. D. Landau, On the theory of slow combustion, Acta Physicochim. URSS 19 (1) (1948) 77-85.

[27] G. H. Markstein, Nonsteady flame propagation, Tech. rep., Pergamon Press (1964).

[28] P. G. Saffman, S. G. Taylor, The penetration of a fluid into a porous medium or Hele-Shaw cell containing a more viscous liquid, Proc. Roy. Soc. London, A 245 (1958) 312-329.

[29] G. I. Sivashinsky, Diffusional-thermal theory of cellular flames, Combust. Sci. Technol. 15 (1977) 137146.

[30] S. H. Kang, S. W. Baek, H. G. Im, Effects of heat and momentum losses on the stability of premixed flames in a narrow channel, Combust. Theory Modelling 10 (4) (2006) 659-681.

[31] G. Joulin, G. I. Sivashinsky, Influence of momentum and heat losses on the large-scale stability of quasi-2D premixed flames, Combust. Sci. Technol. 98 (1994) 11-23.

[32] S. H. Kang, H. G. Im, S. W. Baek, A computational study of Saffman-Taylor instability in premixed flames, Combust. Theory Modelling 7 (2003) 343-363.

[33] S. Kadowaki, H. Suzuki, H. Kobayashi, The unstable behavior of cellular premixed flames induced by intrinsic instability, Proc. Combust. Inst. 30 (2005) 169-176.

[34] C. Altantzis, C. E. Frouzakis, A. G. Tomboulides, M. Matalon, Hydrodynamic and thermodiffusive instability effects on the evolution of laminar planar lean premixed hydrogen flames, J. Fluid Mech. 700 (2012) 329-361.

[35] C. E. Frouzakis, N. Fogla, A. F. Tomboulides, C. Altantzis, M. Matalon, Numerical study of unstable hydrogen/air flames: Shape and propagation speed, Proc. Combust. Inst. 35 (2014) 1087-1095.

[36] Y. Rastigejev, M. Matalon, Nonlinear evolution of hydrodynamically unstable premixed flames, J. Fluid Mech. 554 (2006) 371-392.

[37] A. Matsugi, H. Terashima, Diffusive-thermal effect on local chemical structures in premixed hydrogenair flames, Combust. Flame 179 (2017) 238-241.

[38] J. Wongwiwat, J. Gross, P. D. Ronney, Flame propagation in narrow channels at varying Lewis number, Tech. rep., 25th International Colloquium on the Dynamics of Explosions and Reactive Systems, Paper No. 258 (2015).

[39] J. Sharif, M. Abid, P. D. Ronney, Premixed-gas flame propagation in Hele-Shaw cells, Tech. rep., Spring Technical Meeting, joint U.S. Sections, Combustion Institute (1999).

[40] C. Almarcha, J. Quinard, B. Denet, E. Al-Sharraf, J. Laugier, E. Villermaux, Experimental two dimensional cellular flames, Phys. Fluids 27 (2015) 91110-91111.

[41] J. Jarosinski, Flame quenching by a cold wall, Combust. Flame 50 (1983) 167-175.

[42] P. García-Ybarra, C. Nicoli, P. Clavin, Soret and dilution effects on premixed flames, Combust. Sci. Technol. 42 (1984) 87-109.

[43] E. Fernández-Tarrazo, A. L. Sánchez, A. Liñán, F. A. Williams, The structure of lean hydrogen-air flame balls, Proc. Combust. Inst. 33 (2011) 1203-1210.

[44] M. D. Smooke, V. Giovangigli, Formulation of the premixed and non-premixed test problems, in: M. D. Smooke (Ed.), Reduced Kinetic Mechanisms and Asymptotic Approximations for Methane-Air Flames, Vol. 384 of Lecture Notes in Physics, Springer-Verlag, 1991, pp. 1-28.

[45] L. Kagan, P. Gordon, G. Sivashinsky, An asymptotic study of the transition from slow to fast burning in narrow channels, Proc. Combust. Inst. 35 (2015) 913-920.

[46] Y. Notay, AGMG software and documentation, http://homepages.ulb.ac.be/ ynotay/AGMG.

[47] Y. Notay, An aggregation-based algebraic multigrid method, Electron. Trans. Numer. Anal. 37 (2010) 123-146. 
[48] A. Napov, Y. Notay, An algebraic multigrid method with guaranteed convergence rate, SIAM J. Sci. Comput. 34 (2012) A1079-A1109.

[49] Y. Notay, Aggregation-based algebraic multigrid for convection-diffusion equations, SIAM J. Sci. Comput. 34 (2012) A2288-A2316.

[50] G. J. Sharpe, S. A. E. G. Falle, Nonlinear cellular instabilities of planar premixed flames: numerical simulations of the Reactive Navier-Stokes equations, Combust. Theory Modelling 10 (3) (2006) 483-514.

[51] Y. B. Zel'dovich, A. G. Istratov, N. I. Kidin, V. B. Librovich, Flame propagation in tubes: hydrodynamics and stability, Combust. Sci. Technol. 24 (1980) 1-13. 